\title{
Approximate nonlinear filtering by projection on exponential manifolds of densities
}

\author{
DAMIANO BRIGO ${ }^{1}$, BERNARD HANZON ${ }^{2}$ and FRANÇOIS LE GLAND 3 \\ ${ }^{1}$ Product Development Group, Banca IMI, San Paolo-IMI Group, Corso Matteotti 6, 20121 \\ Milano, Italy.e-mail: brigo@bimimi.it \\ ${ }^{2}$ Department of Econometrics, Free University Amsterdam, De Boelelaan 1105, $1081 \mathrm{HV}$ \\ Amsterdam, The Netherlands.e-mail: bhanzon@econ.vu.nl \\ ${ }^{3}$ Institut National de Recherche en Informatique et en Automatique, Campus de Beaulieu, 35042 \\ Rennes Cédex, France.e-mail: legland@irisa.fr
}

This paper introduces in detail a new systematic method to construct approximate finite-dimensional solutions for the nonlinear filtering problem. Once a finite-dimensional family is selected, the nonlinear filtering equation is projected in Fisher metric on the corresponding manifold of densities, yielding the projection filter for the chosen family. The general definition of the projection filter is given, and its structure is explored in detail for exponential families. Particular exponential families which optimize the correction step in the case of discrete-time observations are given, and an $a$ posteriori estimate of the local error resulting from the projection is defined. Simulation results comparing the projection filter and the optimal filter for the cubic sensor problem are presented. The classical concept of assumed density filter (ADF) is compared with the projection filter. It is shown that the concept of ADF is inconsistent in the sense that the resulting filters depend on the choice of a stochastic calculus, i.e. the Itô or the Stratonovich calculus. It is shown that in the context of exponential families, the projection filter coincides with the Stratonovich-based ADF. An example is provided, which shows that this does not hold in general, for non-exponential families of densities.

Keywords: assumed density filter; differential geometry and statistics; exponential family; finitedimensional filter; Fisher metric; Hellinger metric; nonlinear filtering; projection filter; Stratonovich stochastic differential equations

\section{Introduction}

The filtering problem consists in estimating the state of a stochastic system from noisy observations. More specifically, we consider here the situation where the state evolves according to a stochastic differential equation (SDE), and the objective is to estimate the state from nonlinear observations in additive Gaussian white noise. In the linear Gaussian case the solution consists of the Kalman filter, a finite-dimensional algorithm which computes the first two conditional moments of the state given the observations. Such an algorithm provides also the whole conditional density of the state given the observations, since in the linear case this 
conditional density is Gaussian and hence characterized by the first two moments. In the general nonlinear case, the filtering problem consists in calculating the whole conditional density, which results in an infinite-dimensional filter. Under some regularity conditions, the conditional density exists and is the solution of the Kushner-Stratonovich equation, a stochastic partial differential equation (PDE). In order to avoid infinite dimensionality, some approximation schemes have been proposed, yielding finite-dimensional filters for the unobserved state. A well-known approximation method is the extended Kalman filter (EKF). The EKF is based upon linearization of the state equation around the current estimate, and application of the Kalman filter to the resulting linearized state equation. This procedure finds its justification in heuristic considerations, and not much is known about its performance, except in the case of small observation noise (Picard 1986; 1991; 1993).

Another approximation method in the nonlinear case is the assumed density filter (ADF), obtained from the selection of a few moment equations, which are closed under the assumption that the density is of a certain form, e.g. Gaussian. We present a detailed definition of the assumed density filters in Section 7. However, the ADF can be dangerous from a mathematical point of view. Logical inconsistency of such a procedure is clear, since a false hypothesis can lead to any conclusion. This inconsistency manifests itself when one compares the assumed density filter obtained by using the Itô calculus with the assumed density filter obtained by using the Stratonovich calculus instead. We present an example which shows that the Stratonovich-based ADF and the Itô-based ADF are not directly related by Itô-Stratonovich transformations, i.e. the Stratonovich-based ADF is not just a Stratonovich version of the Itô-based ADF.

Hanzon (1987) introduced the projection filter, which is a finite-dimensional approximate nonlinear filter based on the differential geometric approach to statistics. Brigo et al. (1995a, b; 1998) particularized the projection filter to exponential families in the framework of SDEs on manifolds. In the present paper we introduce the projection filter with full mathematical detail and define it for general families of probability densities. The projection filter is defined by orthogonally projecting the right-hand side of the Kushner-Stratonovich equation onto the tangent space of a finite-dimensional manifold of probability densities, according to the Fisher metric and its extension to the infinite-dimensional space of square roots of densities, known as the Hellinger distance. We then particularize the projection filter to exponential families, which seem to have a privileged role. Indeed, the filtering algorithm can be divided in two parts: the prediction and the correction. The correction part can be made exact by choosing a suitable exponential family defined in terms of the observation function of the given problem. This also simplifies the evaluation of the local error involved in the projection. These advantages of choosing exponential families are confirmed by simulation results for the cubic sensor problem, when comparing an approximation of the optimal filter, based on discretization with a few hundred grid points, with the projection filter for an exponential family with four parameters. The projection filters for exponential families turn out to be related to the assumed density filters described above. This relationship was first given in 1991, when it was proven formally by Hanzon and Hut (1991) that, if one projects orthogonally onto the tangent space of the finitedimensional manifold of Gaussian densities, the resulting projection filter coincides with the Stratonovich-based Gaussian assumed density filter. The performance of this filter has been 
recently studied by Brigo $(1995 ; 1996 b)$ in the case of small observation noise. In the present paper we give a full proof of the above-mentioned equivalence (see also Brigo et al. $(1996 a, b))$. In fact a much more general result will be shown, namely that the projection filter coincides with the Stratonovich-based ADF for any exponential family. As a consequence the projection filter for exponential families can be obtained as a Stratonovichbased ADF, and the filter formulae can be obtained easily from the moment equations. At the same time this equivalence yields a remedy to the lack of logical consistency involved in the definition of the assumed density filters; the Stratonovich-based ADF that updates the moment parameters of an exponential distribution is a well-defined concept, because of its interpretation as a projection filter.

A short description of the contents of the paper is as follows. In Section 2 we give an introduction to the theory of statistical manifolds, and we present some well-known results about exponential families in a geometrical context. The nonlinear filtering problem is presented in Section 3. The projection filter is defined in Section 4 and explored with more detail for exponential families in Section 5. For some convenient exponential families defined in Section 6 an a posteriori estimate of the local error resulting from the projection (the norm of the total projection residual) is given, and simplifying exponential manifolds which yield an exact correction step in the case of discrete time observations are presented. The assumed density filter is introduced in Section 7. We prove the equivalence between $\mathrm{ADF}$ and projection filter for exponential families in Section 8, where we also present an example to show that this equivalence does not hold for general (non-exponential) families. We consider the cubic sensor problem in Section 9, where simulation results are presented for the comparison between the optimal filter and the projection filter. We give conclusions and directions of further research in Section 10.

In this paper the projection in Fisher metric is used as a tool for deriving finitedimensional approximate filters. The same technique can be used for investigated issues on the finite dimensionality of the probability density of diffusion processes. The first results in this direction have been given by Brigo and Pistone (1996) and Brigo (1997).

\section{Statistical manifolds}

On the Euclidean space $\mathbf{R}^{n}$ equipped with its Borel subsets $\mathscr{B}\left(\mathbf{R}^{n}\right)$ we consider a nonnegative and $\sigma$-finite measure $\lambda$, and we define $\mathscr{C} b$ to be the set of all non-negative and finite measures $\mu$ which are absolutely continuous with respect to $\lambda$, and whose density is positive $\lambda$ a.e. For simplicity, we restrict ourselves in this paper to the case where $\lambda$ is the Lebesgue measure on $\mathbf{R}^{n}$. For any density $p$ on $\mathbf{R}^{n}$, the operator $\mathrm{E}_{p}\{\cdot\}$ will denote integration with respect to the measure $p(x) \mathrm{d} x$.

In the following, we denote by $\mathscr{H}:=\{p=\mathrm{d} \mu / \mathrm{d} \lambda: \mu \in \mathscr{C}\}$ the set of all the densities with respect to $\lambda$ of measures contained in $\mathscr{C}$. Note that, as all the measures in $\mathscr{C} b$ are non-negative and finite, we have that, if $p$ is a density in $\mathscr{H}$, then $p \in L_{1}$, and $p^{1 / 2} \in L_{2}$. Since $\mathscr{R}:=\left\{p^{1 / 2}: p \in \mathscr{H}\right\}$ is a subset of $L_{2}$, it is also a metric space, with metric given by the formula $\mathrm{d}\left(p^{1 / 2}, q^{1 / 2}\right):=\left\|p^{1 / 2}-q^{1 / 2}\right\|$, where $\|\cdot\|$ denotes the norm of the Hilbert space $L_{2}$. By using the bijections between $\mathscr{R}, \mathscr{H}$ and $\mathscr{C}$, one obtains in this way a 
metric on $\mathscr{H}$ and $\mathscr{C}$ as well, called the Hellinger metric, and whose square is given by the formula $H^{2}(p, q):=\frac{1}{2}\left\|p^{1 / 2}-q^{1 / 2}\right\|^{2}$ (Jacod and Shiryayev 1987, Chapter IV, Section 1a; Amari 1985, Section 3.5). Note that $\mathscr{B}$ is not locally homeomorphic to $L_{2}$, hence is not a manifold modelled on $L_{2}$.

In the following we give a very quick review of the main concepts we need from differential geometry. For the basic definitions and a more technical introduction on manifolds, tangent vectors and related concepts we refer to Lang (1995), especially for the infinite-dimensional setting, Amari (1985), Murray and Rice (1993), and the references given therein. Consider first an open subset $M$ of $L_{2}$. Let $x$ be a point of $M$, and let $\gamma$ be a curve on $M$ around $x$, i.e. a differentiable map between an open neighbourhood of $0 \in \mathbf{R}$ and $M$ such that $\gamma(0)=x$. We can define the tangent vector to $\gamma$ at $x$ as the Fréchet derivative $D \gamma(0)$, i.e. the linear map defined in $\mathbf{R}$ around 0 and taking values in $L_{2}$ such that the following limit holds:

$$
\lim _{|h| \rightarrow 0} \frac{\|\gamma(h)-\gamma(0)-\mathrm{D} \gamma(0) \cdot h\|}{|h|}=0 .
$$

The map $\mathrm{D} \gamma(0)$ approximates linearly the change of $\gamma$ around $x$. Let $\mathscr{C}_{x}(M)$ be the set of all the curves on $M$ around $x$. If we consider the space (called the tangent space)

$$
L_{x} M:=\left\{\mathrm{D} \gamma(0): \gamma \in \mathscr{C}_{x}(M)\right\}
$$

of tangent vectors to all the possible curves on $M$ around $x$, we obtain again the space $L_{2}$. This is because for every $v \in L_{2}$ we can always consider the straight line $\gamma^{v}(h):=x+h v$. Since $M$ is open, $\gamma^{v}(h)$ takes values in $M$ for $|h|$ small enough. Of course $\mathrm{D} \gamma^{v}(0)=v$, so that indeed $L_{x} M=L_{2}$. Consider next an embedded $m$-dimensional submanifold $N$ of $L_{2}$ (see, for example, Lang (1995, Section II.2) for the definition of a submanifold). For a point $x$ of $N$, we define $L_{x} N$ analogously to $L_{x} M$ :

$$
L_{x} N:=\left\{\mathrm{D} \gamma(0): \gamma \in \mathscr{C}_{x}(N)\right\} .
$$

This is an $m$-dimensional proper linear subspace of $L_{2}$, which is a representation of the tangent space of $N$ at $x$. In our work we shall consider finite-dimensional manifolds $N$ embedded in $L_{2}$, which are contained in $\mathscr{B}$ as a set, i.e. $N \subset \mathscr{R} \subset L_{2}$. As is well known, any manifold may be described by an atlas consisting of charts. For the manifold $N \subset L_{2}$ this means that for any $p^{1 / 2} \in N$ there exists a pair $\left(S^{1 / 2}, \phi\right)$, with $S^{1 / 2}$ an open neighbourhood of $p^{1 / 2}$ in $N$ and $\phi: S^{1 / 2} \rightarrow \Theta$ a homeomorphism of $S^{1 / 2}$ onto an open subset $\Theta$ of $\mathbf{R}^{m}$, such that the inverse map $i$ of $\phi$,

$$
\begin{gathered}
i: \Theta \rightarrow S^{1 / 2} \\
\theta \mapsto\{p(\cdot, \theta)\}^{1 / 2}
\end{gathered}
$$

is a differentiable mapping of $\Theta$ into $L_{2}$, with the property that the derivative $\operatorname{Di}(\theta)$, considered as a linear mapping from $\mathbf{R}^{m}$ to $L_{2}$, is injective at each point $\theta \in \Theta$. Of course the range of $\operatorname{Di}(\theta)$ is precisely $L_{\{p(\cdot \theta)\}^{1 / 2}} N$, and the image of $\Theta$ under $i$ is precisely $S^{1 / 2}$. 


\subsection{General manifolds}

We shall denote by $S$ the following family of probability densities:

$$
S=\{p(\cdot, \theta), \theta \in \Theta\},
$$

where $\Theta \subseteq \mathbf{R}^{m}$ and we shall work only with the single coordinate chart $\left(S^{1 / 2}, \phi\right)$ in the same way as Amari (1985). From the fact that $\left(S^{1 / 2}, \phi\right)$ is a chart, it follows that

$$
\left\{\frac{\partial i(\cdot, \theta)}{\partial \theta_{1}}, \ldots, \frac{\partial i(\cdot, \theta)}{\partial \theta_{m}}\right\}
$$

is a set of linearly independent vectors in $L_{2}$. In such a context, let us see what the vectors of $L_{\{p(\cdot, \theta)\}^{1 / 2}} S^{1 / 2}$ are. We can consider a curve in $S^{1 / 2}$ around $\{p(\cdot, \theta)\}^{1 / 2}$ to be of the form $\gamma: h \mapsto\{p(\cdot, \theta(h))\}^{1 / 2}$, where $h \mapsto \theta(h)$ is a curve in $\Theta$ around $\theta$. Then, according to the chain rule, we compute the following Fréchet derivative:

$$
\mathrm{D} \gamma(0)=\left.\mathrm{D}\{p(\cdot, \theta(h))\}^{1 / 2}\right|_{h=0}=\sum_{i=1}^{m} \frac{\partial\{p(\cdot, \theta)\}^{1 / 2}}{\partial \theta_{i}} \dot{\theta}_{i}(0) .
$$

We obtain that the tangent vector space at $\{p(\cdot, \theta)\}^{1 / 2}$ to the space $S^{1 / 2}$ of square roots of densities of $S$ is given by

$$
L_{\{p(\cdot \theta)\}^{1 / 2}} S^{1 / 2}=\operatorname{span}\left\{\frac{\partial\{p(\cdot, \theta)\}^{1 / 2}}{\partial \theta_{1}}, \ldots, \frac{\partial\{p(\cdot, \theta)\}^{1 / 2}}{\partial \theta_{m}}\right\} .
$$

As $i$ is the inverse of a chart, these vectors are actually linearly independent, and they indeed form a basis of the tangent vector space. One has to be careful because, if this were not true, the dimension of the above spanned space could drop. From now on we assume that indeed $S^{1 / 2}$ is a chart of an $m$-dimensional manifold $N$, so that the tangent vectors in (1) are linearly independent vectors in $L_{2}$. The inner product of any two basis elements is defined, according to the $L_{2}$ inner product

$$
\left\langle\frac{\partial\{p(\cdot, \theta)\}^{1 / 2}}{\partial \theta_{i}}, \frac{\partial\{p(\cdot, \theta)\}^{1 / 2}}{\partial \theta_{j}}\right\rangle=\frac{1}{4} \int \frac{1}{p(x, \theta)} \frac{\partial p(x, \theta)}{\partial \theta_{i}} \frac{\partial p(x, \theta)}{\partial \theta_{j}} \mathrm{~d} x=\frac{1}{4} g_{i j}(\theta) .
$$

This is, up to the numeric factor $\frac{1}{4}$, the Fisher information metric (Amari 1985, Section 2.3; Murray and Rice 1993, Section 6.2). The matrix $g(\theta)=\left(g_{i j}(\theta)\right)$ is called the Fisher information matrix.

Amari (1985, Section 2.3) used a different representation for tangent vectors to $S$ at $p$ and defined an isomorphism between the actual tangent space and the vector space

$$
\operatorname{span}\left\{\frac{\partial \log p(\cdot, \theta)}{\partial \theta_{1}}, \ldots, \frac{\partial \log p(\cdot, \theta)}{\partial \theta_{m}}\right\},
$$

and, on this representation of the tangent space, he defined a Riemannian metric given by

$$
\mathrm{E}_{p(\cdot \theta)}\left\{\frac{\partial \log p(\cdot, \theta)}{\partial \theta_{i}} \frac{\partial \log p(\cdot, \theta)}{\partial \theta_{j}}\right\} .
$$


This is again the Fisher information metric, and indeed this is the most frequent definition of the Fisher metric.

Next, we introduce the orthogonal projection between $L_{2}$ and the finite-dimensional tangent vector space (1). Let us recall that our basis is not orthogonal, so that we have to project according to the following formula:

$$
\begin{gathered}
\Pi: L_{2} \rightarrow \operatorname{span}\left\{w_{1}, \ldots, w_{m}\right\} \\
v \mapsto \sum_{i=1}^{m}\left(\sum_{j=1}^{m} W^{i j}\left\langle v, w_{j}\right\rangle\right) w_{i}
\end{gathered}
$$

where $\left\{w_{1}, \ldots, w_{m}\right\}$ are $m$ linearly independent vectors, $W:=\left(\left\langle w_{i}, w_{j}\right\rangle\right)$ is the matrix formed by all the possible inner products of such linear independent vectors, and $\left(W^{i j}\right)$ is the inverse of the matrix $W$. In our context $\left\{w_{1}, \ldots, w_{m}\right\}$ are the vectors in (1), and of course $W$ is, up to the numeric factor $\frac{1}{4}$, the Fisher information matrix given by (2). Then we obtain the following projection formula, where $\left(g^{i j}(\theta)\right)$ is the inverse of the Fisher information matrix $\left(g_{i j}(\theta)\right)$ :

$$
\begin{aligned}
& \Pi_{\theta}: L_{2} \rightarrow L_{\{p(\cdot, \theta)\}^{1 / 2}} S^{1 / 2}=\operatorname{span}\left\{\frac{\partial\{p(\cdot, \theta)\}^{1 / 2}}{\partial \theta_{1}}, \ldots, \frac{\partial\{p(\cdot, \theta)\}^{1 / 2}}{\partial \theta_{m}}\right\} \\
& v \mapsto \sum_{i=1}^{m}\left(\sum_{j=1}^{m} 4 g^{i j}(\theta)\left\langle v, \frac{\partial\{p(\cdot, \theta)\}^{1 / 2}}{\partial \theta_{j}}\right\rangle\right) \frac{\partial\{p(\cdot, \theta)\}^{1 / 2}}{\partial \theta_{i}} .
\end{aligned}
$$

For elements in $L_{2}$ of the special form given below, the following useful expressions are obtained for the projection, and for the norm of the projection error.

Lemma 2.1. If the function $u$ satisfies

$$
\mathrm{E}_{p(\cdot, \theta)}\left\{|u|^{2}\right\}<\infty
$$

then $v:=\frac{1}{2}\{p(\cdot, \theta)\}^{1 / 2} u$ belongs to $L_{2}$, its projection onto the tangent space $L_{\{p(\cdot, \theta)\}^{1 / 2}} S^{1 / 2}$ is given by

$$
\Pi_{\theta} v=\sum_{i=1}^{m}\left(\sum_{j=1}^{m} g^{i j}(\theta) \mathrm{E}_{p(\cdot, \theta)}\left\{u \frac{\partial \log p(\cdot, \theta)}{\partial \theta_{j}}\right\}\right) \frac{\partial\{p(\cdot, \theta)\}^{1 / 2}}{\partial \theta_{i}},
$$

and the norm of the projection error satisfies

$$
\left\|v-\Pi_{\theta} v\right\|^{2}=\frac{1}{4} \mathrm{E}_{p(\cdot, \theta)}\left\{|u|^{2}\right\}-\frac{1}{4}\left[\mathrm{E}_{p(\cdot, \theta)}\{u \mathrm{D} \log p(\cdot, \theta)\}\right]^{\mathrm{T}}\{g(\theta)\}^{-1} \mathrm{E}_{p(\cdot ; \theta)}\{u \mathrm{D} \log p(\cdot, \theta)\},
$$

where for all $\theta \in \Theta$

$$
\mathrm{D} \log p(\cdot, \theta):=\left[\frac{\partial \log p(\cdot, \theta)}{\partial \theta_{1}}, \ldots, \frac{\partial \log p(\cdot, \theta)}{\partial \theta_{m}}\right]^{\mathrm{T}} .
$$

Proof. Obviously 


$$
\|v\|^{2}=\left\langle\frac{1}{2}\{p(\cdot, \theta)\}^{1 / 2} u, \frac{1}{2}\{p(\cdot, \theta)\}^{1 / 2} u\right\rangle=\frac{1}{4} \mathrm{E}_{p(\cdot, \theta)}\left\{|u|^{2}\right\},
$$

hence $v$ belongs to $L_{2}$. For $j=1, \ldots, m$

$$
\left\langle v, \frac{\partial\{p(\cdot, \theta)\}^{1 / 2}}{\partial \theta_{j}}\right\rangle=\left\langle\frac{1}{2}\{p(\cdot, \theta)\}^{1 / 2} u, \frac{1}{2}\{p(\cdot, \theta)\}^{1 / 2} \frac{\partial \log p(\cdot, \theta)}{\partial \theta_{j}}\right\rangle=\frac{1}{4} \mathrm{E}_{p(\cdot, \theta)}\left\{u \frac{\partial \log p(\cdot, \theta)}{\partial \theta_{j}}\right\},
$$

and substitution into (3) yields (4). Finally, the vectors $v-\Pi_{\theta} v$ and $\Pi_{\theta} v$ are orthogonal; hence

$$
\left\|v-\Pi_{\theta} v\right\|^{2}=\|v\|^{2}-\left\langle v, \Pi_{\theta} v\right\rangle
$$

which yields (5).

\subsection{Manifolds associated with exponential families}

We conclude this section with some well-known results about exponential families, which will be used in the following sections. More results on exponential families have been given by Amari (1985, Chapter 4) and by Barndorff-Nielsen (1978). Although the definition of an exponential family can be given for an arbitrary dominating measure $\lambda$, we restrict ourselves to the case where $\lambda$ is the Lebesgue measure on $\mathbf{R}^{n}$. The reason for doing so is that, in most of the filtering literature (see, for example, Davis and Marcus (1981), Pardoux (1991) and Rozovskii (1990)), the conditional probability distributions are absolutely continuous with respect to the Lebesgue measure, and the filtering equations, such as (10) below, are stated for the conditional density with respect to the Lebesgue measure. We shall use the following equivalent notation for partial differentiation:

$$
\frac{\partial^{k}}{\partial \theta_{i_{1}} \ldots \partial \theta_{i_{k}}}=\partial_{i_{1}, \ldots, i_{k}}^{k} .
$$

Definition 2.2. Let $\left\{c_{1}, \ldots, c_{m}\right\}$ be scalar measurable functions defined on $\mathbf{R}^{n}$, such that $\left\{1, c_{1}, \ldots, c_{m}\right\}$ are linearly independent, and assume that the convex set

$$
\Theta_{0}:=\left\{\theta \in \mathbf{R}^{m}: \psi(\theta)=\log \left(\int \exp \left\{\theta^{\mathrm{T}} c(x)\right\} \mathrm{d} x\right)<\infty\right\},
$$

has a non-empty interior. Then

$$
\operatorname{EM}(c):=\{p(\cdot, \theta), \theta \in \Theta\}, \quad p(x, \theta):=\exp \left\{\theta^{\mathrm{T}} c(x)-\psi(\theta)\right\},
$$

where $\Theta \subseteq \Theta_{0}$ is open, is called an exponential family of probability densities.

Throughout the paper, when using the notation $\operatorname{EM}(c)$, it is assumed that the coefficients $\left\{c_{1}, \ldots, c_{m}\right\}$ satisfy the assumptions in Definition 2.2.

Lemma 2.3. Consider the exponential family $\operatorname{EM}(c)$. The function $\psi$ is infinitely differentiable in $\Theta$ : 


$$
\begin{gathered}
\mathrm{E}_{p(\cdot, \theta)}\left\{c_{i}\right\}=\partial_{i} \psi(\theta)=: \eta_{i}(\theta), \\
\mathrm{E}_{p(\cdot, \theta)}\left\{c_{i} c_{j}\right\}=\partial_{i j}^{2} \psi(\theta)+\partial_{i} \psi(\theta) \partial_{j} \psi(\theta),
\end{gathered}
$$

and more generally

$$
\mathrm{E}_{p(\cdot, \theta)}\left\{c_{i_{1}} \ldots c_{i_{k}}\right\}=\exp \{-\psi(\theta)\} \frac{\partial^{k}}{\partial \theta_{i_{1}} \ldots \partial \theta_{i_{k}}} \exp \{\psi(\theta)\} .
$$

The Fisher information matrix satisfies

$$
g_{i j}(\theta)=\partial_{i j}^{2} \psi(\theta)=\partial_{i} \eta_{j}(\theta) .
$$

Remark 2.4. The quantities

$$
\left(\eta_{1}, \ldots, \eta_{m}\right) \in \mathscr{E}=\eta(\Theta) \subset \mathbf{R}^{m}
$$

form a coordinate system for the given exponential family. The two coordinate systems $\theta$ (canonical parameters) and $\eta$ (expectation parameters) are related by diffeomorphism, and according to the above results the Jacobian matrix of the transformation $\eta=\eta(\theta)$ is the Fisher information matrix. We shall use the notation $p_{E}(\cdot, \eta)=p(\cdot, \theta)$ to express exponential densities of $\operatorname{EM}(c)$ as functions of the expectation parameters.

An important result of Amari (1985, Section 3.4) is that the canonical parameters and the expectation parameters are biorthogonal with respect to the Fisher information metric; at $\{p(\cdot, \theta)\}^{1 / 2}=\left\{p_{E}(\cdot, \eta)\right\}^{1 / 2}$

$$
\left\langle\frac{\partial}{\partial \theta_{i}}\{p(\cdot, \theta)\}^{1 / 2}, \frac{\partial}{\partial \eta_{j}}\left\{p_{E}(\cdot, \theta)\right\}^{1 / 2}\right\rangle=\frac{1}{4} \delta_{i j}, \quad i, j=1,2, \ldots, m .
$$

All these results have been given by or can be immediately derived from the work of Amari (1985, Chapter 4) or Barndorff-Nielsen (1978, Theorem 8.1).

\section{The nonlinear filtering problem}

On the probability space $(\Omega, \mathscr{F}, \mathbf{P})$ with the filtration $\left\{\mathscr{F}_{t}, t \geqslant 0\right\}$ we consider the following state and observation equations (Jazwinski 1970; Maybeck 1979; Davis and Marcus 1981):

$$
\begin{gathered}
\mathrm{d} X_{t}=f_{t}\left(X_{t}\right) \mathrm{d} t+\sigma_{t}\left(X_{t}\right) \mathrm{d} W_{t}, \quad X_{0}, \\
\mathrm{~d} Y_{t}=h_{t}\left(X_{t}\right) \mathrm{d} t+\mathrm{d} V_{t}, \quad Y_{0}=0 .
\end{gathered}
$$

These equations are Itô SDEs. In (7), the unobserved state process $\left\{X_{t}, t \geqslant 0\right\}$ and the observation process $\left\{Y_{t}, t \geqslant 0\right\}$ are taking values in $\mathbf{R}^{n}$ and $\mathbf{R}^{d}$ respectively; the noise processes $\left\{W_{t}, t \geqslant 0\right\}$ and $\left\{V_{t}, t \geqslant 0\right\}$ are two Brownian motions, taking values in $\mathbf{R}^{p}$ and $\mathbf{R}^{d}$, with covariance matrices $Q_{t}$ and $R_{t}$ respectively. We assume that $R_{t}$ is invertible for all $t \geqslant 0$, which implies, without loss of generality, that we can take $R_{t}=I$ for all $t \geqslant 0$. 
Finally, the initial state $X_{0}$ and the noise processes $\left\{W_{t}, t \geqslant 0\right\}$ and $\left\{V_{t}, t \geqslant 0\right\}$ are mutually independent.

We assume that the initial state $X_{0}$ has a density $p_{0}$ with respect to the Lebesgue measure on $\mathbf{R}^{n}$, and has finite moments of any order, and we make the following assumptions on the coefficients $f_{t}, a_{t}:=\sigma_{t} Q_{t} \sigma_{t}^{\mathrm{T}}$, and $h_{t}$ of the system (7).

(A) Local Lipschitz continuity: for all $R>0$, there exists $K_{R}>0$ such that

$$
\left|f_{t}(x)-f_{t}\left(x^{\prime}\right)\right| \leqslant K_{R}\left|x-x^{\prime}\right| \quad \text { and } \quad\left\|a_{t}(x)-a_{t}\left(x^{\prime}\right)\right\| \leqslant K_{R}\left|x-x^{\prime}\right|,
$$

for all $t \geqslant 0$, and for all $x, x^{\prime} \in B_{R}$, the ball of radius $R$ centred at the origin.

(B) Non-explosion: there exists $K>0$ such that

$$
x^{\mathrm{T}} f_{t}(x) \leqslant K\left(1+|x|^{2}\right) \quad \text { and } \quad\left\|a_{t}(x)\right\| \leqslant K\left(1+|x|^{2}\right),
$$

for all $t \geqslant 0$, and for all $x \in \mathbf{R}^{n}$.

(C) Polynomial growth: there exist $K>0$ and $r \geqslant 0$ such that

$$
\left|h_{t}(x)\right| \leqslant K\left(1+|x|^{r}\right)
$$

for all $t \geqslant 0$, and for all $x \in \mathbf{R}^{n}$.

Under Assumptions (A) and (B), there exists a pathwise-unique solution $\left\{X_{t}, t \geqslant 0\right\}$ to the state equation (Khasminskii 1980, Chapter 3, Theorem 4.1 with the Lyapunov function $V(x)=1+|x|^{2}$ ), and $X_{t}$ has finite moments of any order. Under the additional Assumption (C) the following finite-energy condition holds:

$$
\mathbf{E} \int_{0}^{T}\left|h_{t}\left(X_{t}\right)\right|^{2} \mathrm{~d} t<\infty, \quad \text { for all } T \geqslant 0 .
$$

The nonlinear filtering problem consists in finding the conditional probability distribution $\pi_{t}$ of the state $X_{t}$ given the observations up to time $t$, i.e. $\pi_{t}(\mathrm{~d} x):=\mathbf{P}\left[X_{t} \in \mathrm{d} x \mid \mathscr{H}_{t}\right]$, where $\mathscr{Y}_{t}:=\sigma\left(Y_{s}, 0 \leqslant s \leqslant t\right)$. For a tutorial on nonlinear filtering, see Davis and Marcus (1981) or van Schuppen (1979). Since the finite-energy condition holds, it follows from Fujisaki et al. (1972, Theorem 4.1) or Pardoux (1991, Théorème 2.3.7) that $\left\{\pi_{t}, t \geqslant 0\right\}$ satisfies the Kushner-Stratonovich equation, i.e. for any smooth and compactly supported test function $\phi$ defined on $\mathbf{R}^{n}$

$$
\pi_{t}(\phi)=\pi_{0}(\phi)+\int_{0}^{t} \pi_{s}\left(\mathscr{L}_{s} \phi\right) \mathrm{d} s+\sum_{k=1}^{d} \int_{0}^{t}\left\{\pi_{s}\left(h_{s}^{k} \phi\right)-\pi_{s}\left(h_{s}^{k}\right) \pi_{s}(\phi)\right\}\left\{\mathrm{d} Y_{s}^{k}-\pi_{s}\left(h_{s}^{k}\right) \mathrm{d} s\right\},
$$

where for all $t \geqslant 0$, the backward diffusion operator $\mathscr{B}_{t}$ is defined by

$$
\mathscr{D}_{t}=\sum_{i=1}^{n} f_{t}^{i} \frac{\partial}{\partial x_{i}}+\frac{1}{2} \sum_{i, j=1}^{n} a_{t}^{i j} \frac{\partial^{2}}{\partial x_{i} \partial x_{j}}
$$

and where we set 


$$
\pi_{t}(\phi)=\int \phi(x) \pi_{t}(\mathrm{~d} x)=\mathbf{E}\left[\phi\left(X_{t}\right) \mid \mathscr{Y}_{t}\right]
$$

for the conditional expectation of the random variable $\phi\left(X_{t}\right)$ given the observations up to time $t$. The Stratonovich form of (8) is obtained, after straightforward computations, as

$$
\begin{aligned}
\pi_{t}(\phi)= & \pi_{0}(\phi)+\int_{0}^{t} \pi_{s}\left(\mathscr{C}_{s} \phi\right) \mathrm{d} s-\frac{1}{2} \int_{0}^{t}\left\{\pi_{s}\left(\left|h_{s}\right|^{2} \phi\right)-\pi_{s}\left(\left|h_{s}\right|^{2}\right) \pi_{s}(\phi)\right\} \mathrm{d} s \\
& +\sum_{k=1}^{d} \int_{0}^{t}\left\{\pi_{s}\left(h_{s}^{k} \phi\right)-\pi_{s}\left(h_{s}^{k}\right) \pi_{s}(\phi)\right\} \circ \mathrm{d} Y_{s}^{k},
\end{aligned}
$$

where, here and throughout the paper, the symbol $\circ$ denotes a Stratonovich integral. For all $t \geqslant 0$, the probability distribution $\pi_{t}$ has a density $p_{t}$ with respect to the Lebesgue measure on $\mathbf{R}^{n}$, which satisifes

$$
\mathrm{d} p_{t}=\mathscr{L}_{t}^{*} p_{t} \mathrm{~d} t+\sum_{k=1}^{d} p_{t}\left(h_{t}^{k}-\mathrm{E}_{p_{t}}\left\{h_{t}^{k}\right\}\right)\left(\mathrm{d} Y_{t}^{k}-\mathrm{E}_{p_{t}}\left\{h_{t}^{k}\right\} \mathrm{d} t\right),
$$

where $\mathrm{E}_{p_{t}}\{\cdot\}$ denotes the expectation with respect to the probability density $p_{t}$, i.e. the conditional expectation given the observations up to time $t$, and where for all $t \geqslant 0$, the forward diffusion operator $\mathscr{L}_{t}^{*}$ is defined by

$$
\mathscr{D}_{t}^{*} \phi=-\sum_{i=1}^{n} \frac{\partial}{\partial x_{i}}\left[f_{t}^{i} \phi\right]+\frac{1}{2} \sum_{i, j=1}^{n} \frac{\partial^{2}}{\partial x_{i} \partial x_{j}}\left[a_{t}^{i j} \phi\right],
$$

for any test function $\phi$ defined on $\mathbf{R}^{n}$; see Pardoux (1991) for precise statements. The corresponding Stratonovich form of (10) is

$$
\mathrm{d} p_{t}=\mathscr{B}_{t}^{*} p_{t} \mathrm{~d} t-\frac{1}{2} p_{t}\left[\left|h_{t}\right|^{2}-\mathrm{E}_{p_{t}}\left\{\left|h_{t}\right|^{2}\right\}\right] \mathrm{d} t+\sum_{k=1}^{d} p_{t}\left(h_{t}^{k}-\mathrm{E}_{p_{t}}\left\{h_{t}^{k}\right\}\right) \circ \mathrm{d} Y_{t}^{k} .
$$

As explained in Section 2, we shall work with the square roots of densities, rather than the densities themselves. Using the Stratonovich chain rule, we obtain that $\left\{p_{t}^{1 / 2}, t \geqslant 0\right\}$ satisfies

$$
\begin{aligned}
\mathrm{d} p_{t}^{1 / 2} & =\frac{1}{2 p_{t}^{1 / 2}} \circ \mathrm{d} p_{t}=\frac{1}{2} p_{t}^{1 / 2} \alpha_{t}\left(p_{t}\right) \mathrm{d} t-\frac{1}{2} p_{t}^{1 / 2} \beta_{t}^{0}\left(p_{t}\right) \mathrm{d} t+\frac{1}{2} \sum_{k=1}^{d} p_{t}^{1 / 2} \beta_{t}^{k}\left(p_{t}\right) \circ \mathrm{d} Y_{t}^{k} \\
& =\mathscr{P}_{t}\left(p_{t}^{1 / 2}\right) \mathrm{d} t-Q_{t}^{0}\left(p_{t}^{1 / 2}\right) \mathrm{d} t+\sum_{k=1}^{d} Q_{t}^{k}\left(p_{t}^{1 / 2}\right) \circ \mathrm{d} Y_{t}^{k},
\end{aligned}
$$

where the nonlinear time-dependent operators $\mathscr{P}_{t}$ and $Q_{t}^{k}$ for $k=0,1, \ldots, d$ are defined by 


$$
\mathscr{P}_{t}\left(p^{1 / 2}\right):=\frac{1}{2} p^{1 / 2} \alpha_{t}(p), \quad Q_{t}^{k}\left(p^{1 / 2}\right):=\frac{1}{2} p^{1 / 2} \beta_{t}^{k}(p),
$$

respectively and

$$
\alpha_{t}(p):=\frac{\mathscr{B}_{t}^{*} p}{p}, \quad \beta_{t}^{0}(p):=\frac{1}{2}\left[\left|h_{t}\right|^{2}-\mathrm{E}_{p}\left\{\left|h_{t}\right|^{2}\right\}\right], \quad \beta_{t}^{k}(p):=h_{t}^{k}-\mathrm{E}_{p}\left\{h_{t}^{k}\right\},
$$

for $k=1, \ldots, d$. Simple calculations show that

$$
\begin{aligned}
\alpha_{t}(p)= & -\sum_{i=1}^{n}\left(f_{t}^{i} \frac{\partial}{\partial x_{i}}(\log p)+\frac{\partial f_{t}^{i}}{\partial x_{i}}\right) \\
& +\frac{1}{2} \sum_{i, j=1}^{n}\left(a_{t}^{i j} \frac{\partial^{2}}{\partial x_{i} \partial x_{j}}(\log p)+a_{t}^{i j} \frac{\partial}{\partial x_{i}}(\log p) \frac{\partial}{\partial x_{j}}(\log p)+2 \frac{\partial a_{t}^{i j}}{\partial x_{j}} \frac{\partial}{\partial x_{i}}(\log p)+\frac{\partial^{2} a_{t}^{i j}}{\partial x_{i} \partial x_{j}}\right) .
\end{aligned}
$$

\section{General definition of the projection filter}

In the present section we shall introduce the general definition of the projection filter. We begin by noting that the stochastic calculus to be used in this derivation is the Stratonovich calculus. This is a standard choice for stochastic calculus on manifolds, as one can see for example in Elworthy (1982), and is due to difficulties in interpreting second-order terms arising in the Itô calculus in terms of manifold structures. This choice can be further motivated by the following example.

Example 4.1. Consider the two-dimensional SDEs with the initial condition $\left(X_{0}, Y_{0}\right)=(0,0)$ given by

$$
\mathrm{d}\left[\begin{array}{c}
X_{t} \\
Y_{t}
\end{array}\right]=\left[\begin{array}{c}
1 \\
2 X_{t}
\end{array}\right] \mathrm{d} W_{t} \quad \text { and } \quad \mathrm{d}\left[\begin{array}{c}
X_{t} \\
Y_{t}
\end{array}\right]=\left[\begin{array}{c}
1 \\
2 X_{t}
\end{array}\right] \circ \mathrm{d} W_{t} .
$$

Note that the vector field on the right-hand side of both equations is tangent to the parabola $\mathscr{P}:=\left\{(x, y) \in \mathbf{R}^{2}: y=x^{2}\right\}$ and that $\left(X_{0}, Y_{0}\right)$ belongs to $\mathscr{P}$, so that one would expect the solution to stay in $\mathscr{P}$ for all times. However, it is easy to check that the solutions of the above equations are

$$
\left[\begin{array}{c}
W_{t} \\
W_{t}^{2}-t
\end{array}\right] \quad \text { and } \quad\left[\begin{array}{c}
W_{t} \\
W_{t}^{2}
\end{array}\right]
$$

respectively, i.e. the solution of the equation in the Stratonovich sense stays in $\mathscr{P}$ for all times, whereas the solution of the equation in the Itô sense does not. It is therefore intuitive that, if one projects the vector fields of a SDE written in the Ito form onto the tangent space of a manifold, then the solution of the resulting equation would in general leave the manifold. 
This cannot happen if one projects the vector fields of the same SDE written in the Stratonovich sense.

We shall assume that the finite-dimensional manifold $S^{1 / 2}$ that we are working with has a manifold structure and a well-defined Fisher information metric at all points $\theta \in \Theta$, according to the presentation given in Section 2. In order to project the KushnerStratonovich equation for $p_{t}^{1 / 2}$ given in Section 3 onto the $m$-dimensional manifold $S^{1 / 2}$ we require the following assumption to be satisfied:

(D) For all $\theta \in \Theta$

$$
\sup _{t \geqslant 0} \mathrm{E}_{p(\cdot, \theta)}\left\{\left|\frac{\mathscr{L}_{t}^{*} p(\cdot, \theta)}{p(\cdot, \theta)}\right|^{2}\right\}<\infty \quad \text { and } \quad \sup _{t \geqslant 0} \mathrm{E}_{p(\cdot, \theta)}\left\{\left|h_{t}\right|^{4}\right\}<\infty .
$$

This assumption will be explored in detail for exponential families in Section 5, and explicit sufficient conditions under which it holds will be given. This assumption ensures that, for all $\theta \in \Theta$ and all $t \geqslant 0$, the vectors $\mathscr{P}_{t}\left(\{p(\cdot, \theta)\}^{1 / 2}\right)$ and $\mathscr{Q}_{t}^{k}\left(\{p(\cdot, \theta)\}^{1 / 2}\right)$ for $k=0,1, \ldots, d$ are vectors in $L_{2}$, so that indeed the projection can take place according to the $L_{2}$ structure described in Section 2.

The projection filter for the family $S=\{p(\cdot, \theta), \theta \in \Theta\}$ is defined as the solution of the following $\mathrm{SDE}$ on the manifold $S^{1 / 2}$ :

$$
\begin{aligned}
\mathrm{d}\left\{p\left(\cdot, \theta_{t}\right)\right\}^{1 / 2}= & \Pi_{\theta_{t}} \circ \mathscr{P}_{t}\left(\left\{p\left(\cdot, \theta_{t}\right)\right\}^{1 / 2}\right) \mathrm{d} t-\Pi_{\theta_{t}} \circ \mathscr{Q}_{t}^{0}\left(\left\{p\left(\cdot, \theta_{t}\right)\right\}^{1 / 2}\right) \mathrm{d} t \\
& +\sum_{k=1}^{d} \Pi_{\theta_{t}} \circ \mathcal{Q}_{t}^{k}\left(\left\{p\left(\cdot, \theta_{t}\right)\right\}^{1 / 2}\right) \circ \mathrm{d} Y_{t}^{k},
\end{aligned}
$$

where for all $\theta \in \Theta$, the projection map $\Pi_{\theta}$ is defined in (3).

Remark 4.2. Although at first sight (15) may look like a stochastic PDE, it is just a finitedimensional SDE which can be equivalently written using different coordinates as an equation in $\Theta \subset \mathbf{R}^{m}$ for the parameter $\theta_{t}$. The explicit form of this SDE is given in the following theorem.

Theorem 4.3. Assume that, in addition to satisfying (A)-(D) the coefficients $f_{t}, a_{t}$ and $h_{t}$ of the system (7), and the family $S$ are such that the maps

$$
\theta \mapsto \mathrm{E}_{p(\cdot, \theta)}\left\{\frac{\mathscr{B}_{t}^{*} p(\cdot, \theta)}{p(\cdot, \theta)} \mathrm{D} \log p(\cdot, \theta)\right\}, \quad \theta \mapsto \mathrm{E}_{p(\cdot, \theta)}\left\{\frac{1}{2}\left|h_{t}\right|^{2} \mathrm{D} \log p(\cdot, \theta)\right\}
$$

and

$$
\theta \mapsto \mathrm{E}_{p(\cdot, \theta)}\left\{h_{t}^{k} \mathrm{D} \log p(\cdot, \theta)\right\},
$$

for $k=1, \ldots, d$, are locally Lipschitz continuous in $\Theta$, uniformly in $t \geqslant 0$.

Then, for all $\theta \in \Theta$ and all $t \geqslant 0$ 
(a) the vectors $\mathscr{P}_{t}\left(\{p(\cdot, \theta)\}^{1 / 2}\right)$ and $Q_{t}^{k}\left(\{p(\cdot, \theta)\}^{1 / 2}\right)$ for $k=0,1, \ldots, d$ are vectors in $L_{2}$ and

(b) the nonlinear operators $\Pi_{\theta} \circ \mathscr{P}_{t}$ and $\Pi_{\theta} \circ Q_{t}^{k}$ for $k=0,1, \ldots, d$ are vector fields on the manifold $S^{1 / 2}$.

The projection filter density $p\left(\cdot, \theta_{t}\right)$ is described by (15), and the projection filter parameters satisfy the following SDE:

$$
\begin{aligned}
\mathrm{d} \theta_{t}=\{ & \left.g\left(\theta_{t}\right)\right\}^{-1} \mathrm{E}_{p\left(\cdot, \theta_{t}\right)}\left\{\frac{\mathscr{D}_{t}^{*} p\left(\cdot, \theta_{t}\right)}{p\left(\cdot, \theta_{t}\right)} \mathrm{D} \log p\left(\cdot, \theta_{t}\right)\right\} \mathrm{d} t \\
& -\left\{g\left(\theta_{t}\right)\right\}^{-1} \mathrm{E}_{p\left(\cdot, \theta_{t}\right)}\left\{\frac{1}{2}\left|h_{t}\right|^{2} \mathrm{D} \log p\left(\cdot, \theta_{t}\right)\right\} \mathrm{d} t \\
& +\sum_{k=1}^{d}\left\{g\left(\theta_{t}\right)\right\}^{-1} \mathrm{E}_{p\left(\cdot, \theta_{t}\right)}\left\{h_{t}^{k} \mathrm{D} \log p\left(\cdot, \theta_{t}\right)\right\} \circ \mathrm{d} Y_{t}^{k} .
\end{aligned}
$$

Under the assumptions on the coefficients, this equation has a unique solution up to the a.s. positive exit time $\tau:=\inf \left\{t \geqslant 0: \theta_{t} \notin \Theta\right\}$.

Proof. Let us compute the projections of the vectors on the right-hand side of the KushnerStratonovich equation (11), using the definitions (12) and (13) and the formula (4) (under Assumption (D) such projections always exist):

$$
\begin{aligned}
\Pi_{\theta_{t}} \circ \mathscr{P}_{t}\left(\left\{p\left(\cdot, \theta_{t}\right)\right\}^{1 / 2}\right) & =\Pi_{\theta_{t}}\left[\frac{1}{2}\left\{p\left(\cdot, \theta_{t}\right)\right\}^{1 / 2} \alpha_{t}\left(p\left(\cdot, \theta_{t}\right)\right)\right] \\
& =\sum_{i=1}^{m}\left(\sum_{j=1}^{m} g^{i j}\left(\theta_{t}\right) \mathrm{E}_{p\left(\cdot, \theta_{t}\right)}\left\{\frac{\mathscr{B}_{t}^{*} p\left(\cdot, \theta_{t}\right)}{p\left(\cdot, \theta_{t}\right)} \frac{\partial \log p\left(\cdot, \theta_{t}\right)}{\partial \theta_{j}}\right\}\right) \frac{\partial\left\{p\left(\cdot, \theta_{t}\right)\right\}^{1 / 2}}{\partial \theta_{i}} .
\end{aligned}
$$

Similarly

$$
\begin{aligned}
\Pi_{\theta_{t}} \circ Q_{t}^{0}\left(\left\{p\left(\cdot, \theta_{t}\right)\right\}^{1 / 2}\right)= & \Pi_{\theta_{t}}\left[\frac{1}{2}\left\{p\left(\cdot, \theta_{t}\right)\right\}^{1 / 2} \beta_{t}^{0}\left(p\left(\cdot, \theta_{t}\right)\right)\right] \\
= & \sum_{i=1}^{m}\left(\sum_{j=1}^{m} g^{i j}\left(\theta_{t}\right) \mathrm{E}_{p\left(\cdot, \theta_{t}\right)}\left\{\frac{1}{2}\left[\left|h_{t}\right|^{2}-\mathrm{E}_{p\left(\cdot, \theta_{t}\right)}\left\{\left|h_{t}\right|^{2}\right\}\right] \frac{\partial \log p\left(\cdot, \theta_{t}\right)}{\partial \theta_{j}}\right\}\right) \\
& \times \frac{\partial\left\{p\left(\cdot, \theta_{t}\right)\right\}^{1 / 2}}{\partial \theta_{i}} \\
= & \sum_{i=1}^{m}\left(\sum_{j=1}^{m} g^{i j}\left(\theta_{t}\right) \mathrm{E}_{p\left(\cdot, \theta_{t}\right)}\left\{\frac{1}{2}\left|h_{t}\right|^{2} \frac{\partial \log p\left(\cdot, \theta_{t}\right)}{\partial \theta_{j}}\right\}\right) \frac{\partial\left\{p\left(\cdot, \theta_{t}\right)\right\}^{1 / 2}}{\partial \theta_{i}}
\end{aligned}
$$

and 


$$
\begin{aligned}
\Pi_{\theta_{t}} \circ Q_{t}^{k}\left(\left\{p\left(\cdot, \theta_{t}\right)\right\}^{1 / 2}\right)= & \Pi_{\theta_{t}}\left[\frac{1}{2}\left\{p\left(\cdot, \theta_{t}\right)\right\}^{1 / 2} \beta_{t}^{k}\left(p\left(\cdot, \theta_{t}\right)\right)\right] \\
= & \sum_{i=1}^{m}\left(\sum_{j=1}^{m} g^{i j}\left(\theta_{t}\right) \mathrm{E}_{p\left(\cdot, \theta_{t}\right)}\left\{\left[h_{t}^{k}-\mathrm{E}_{p\left(\cdot, \theta_{t}\right)}\left\{h_{t}^{k}\right\}\right] \frac{\partial \log p\left(\cdot, \theta_{t}\right)}{\partial \theta_{j}}\right\}\right) \\
& \times \frac{\partial\left\{p\left(\cdot, \theta_{t}\right)\right\}^{1 / 2}}{\partial \theta_{i}} \\
= & \sum_{i=1}^{m}\left(\sum_{j=1}^{m} g^{i j}\left(\theta_{t}\right) \mathrm{E}_{p\left(\cdot, \theta_{t}\right)}\left\{h_{t}^{k} \frac{\partial \log p\left(\cdot, \theta_{t}\right)}{\partial \theta_{j}}\right\}\right) \frac{\partial\left\{p\left(\cdot, \theta_{t}\right)\right\}^{1 / 2}}{\partial \theta_{i}}
\end{aligned}
$$

for $k=1, \ldots, d$. We have used the fact that the constant terms $\mathrm{E}_{p\left(\cdot, \theta_{t}\right)}\left\{\left|h_{t}\right|^{2}\right\}$ and $\mathrm{E}_{p\left(\cdot, \theta_{t}\right)}\left\{h_{t}^{k}\right\}$ give no contribution to the projection, since

$$
\mathrm{E}_{p\left(\cdot, \theta_{t}\right)}\left\{\frac{\partial \log p\left(\cdot, \theta_{t}\right)}{\partial \theta_{j}}\right\}=\int \frac{\partial p\left(x, \theta_{t}\right)}{\partial \theta_{j}} \mathrm{~d} x=0
$$

for $j=1, \ldots, m$. We rewrite (15) in the more detailed form

$$
\begin{aligned}
\mathrm{d}\left\{p\left(\cdot, \theta_{t}\right)\right\}^{1 / 2}= & \sum_{i=1}^{m}\left(\sum_{j=1}^{m} g^{i j}\left(\theta_{t}\right) \mathrm{E}_{p\left(\cdot, \theta_{t}\right)}\left\{\frac{\mathscr{L}_{t}^{*} p\left(\cdot, \theta_{t}\right)}{p\left(\cdot, \theta_{t}\right)} \frac{\partial \log p\left(\cdot, \theta_{t}\right)}{\partial \theta_{j}}\right\}\right) \frac{\partial\left\{p\left(\cdot, \theta_{t}\right)\right\}^{1 / 2}}{\partial \theta_{i}} \mathrm{~d} t \\
& -\sum_{i=1}^{m}\left(\sum_{j=1}^{m} g^{i j}\left(\theta_{t}\right) \mathrm{E}_{p\left(\cdot, \theta_{t}\right)}\left\{\frac{1}{2}\left|h_{t}\right|^{2} \frac{\partial \log p\left(\cdot, \theta_{t}\right)}{\partial \theta_{j}}\right\}\right) \frac{\partial\left\{p\left(\cdot, \theta_{t}\right)\right\}^{1 / 2}}{\partial \theta_{i}} \mathrm{~d} t \\
& +\sum_{i=1}^{m} \sum_{k=1}^{d}\left(\sum_{j=1}^{m} g^{i j}\left(\theta_{t}\right) \mathrm{E}_{p\left(\cdot, \theta_{t}\right)}\left\{h_{t}^{k} \frac{\partial \log p\left(\cdot, \theta_{t}\right)}{\partial \theta_{j}}\right\}\right) \frac{\partial\left\{p\left(\cdot, \theta_{t}\right)\right\}^{1 / 2}}{\partial \theta_{i}} \mathrm{~d} Y_{t}^{k} .
\end{aligned}
$$

By expanding $\left\{p\left(\cdot, \theta_{t}\right)\right\}^{1 / 2}$ according to the Stratonovich chain rule

$$
\mathrm{d}\left\{p\left(\cdot, \theta_{t}\right)\right\}^{1 / 2}=\sum_{i=1}^{m} \frac{\partial\left\{p\left(\cdot, \theta_{t}\right)\right\}^{1 / 2}}{\partial \theta_{i}} \circ \mathrm{d} \theta_{t}^{i},
$$

and comparing with (17) we obtain the following equation for the parameters describing our projected density in $S$ : 


$$
\begin{aligned}
\mathrm{d} \theta_{t}^{i}= & \left(\sum_{j=1}^{m} g^{i j}\left(\theta_{t}\right) \mathrm{E}_{p\left(\cdot, \theta_{t}\right)}\left\{\frac{\mathscr{L}_{t}^{*} p\left(\cdot, \theta_{t}\right)}{p\left(\cdot, \theta_{t}\right)} \frac{\partial \log p\left(\cdot, \theta_{t}\right)}{\partial \theta_{j}}\right\}\right) \mathrm{d} t \\
& -\left(\sum_{j=1}^{m} g^{i j}\left(\theta_{t}\right) \mathrm{E}_{p\left(\cdot, \theta_{t}\right)}\left\{\frac{1}{2}\left|h_{t}\right|^{2} \frac{\partial \log p\left(\cdot, \theta_{t}\right)}{\partial \theta_{j}}\right\}\right) \mathrm{d} t \\
& +\sum_{k=1}^{d}\left(\sum_{j=1}^{m} g^{i j}\left(\theta_{t}\right) \mathrm{E}_{p\left(\cdot, \theta_{t}\right)}\left\{h_{t}^{k} \frac{\partial \log p\left(\cdot, \theta_{t}\right)}{\partial \theta_{j}}\right\}\right) \circ \mathrm{d} Y_{t}^{k}
\end{aligned}
$$

for $i=1, \ldots, m$. Writing the above equation in vector form yields (16). Under the assumptions on the coefficients and on the family $S$, this equation has a unique solution up to the almost-surely positive exit time $\tau$ (Khasminskii 1980, Chapter III, Section 4; Kunita 1984, Chapter II, Theorem 5.2).

\section{The exponential projection filter}

In this section we shall consider the projection filter in the special case where $S^{1 / 2}=\mathrm{EM}^{1 / 2}(c)$. A first possible derivation of the exponential projection filter equations is by specializing the results of Theorem 4.3; see the proof of Theorem 5.4 below. Alternatively, we can also remark that in the special case where $S^{1 / 2}=\operatorname{EM}^{1 / 2}(c)$, and under the same Assumption (D) already introduced in the previous section, it is possible to define for all $\theta \in \Theta$ and all $t \geqslant 0$ a larger but finite-dimensional (smoothly embedded) submanifold $\Sigma_{t, \theta}^{1 / 2}$ of $L_{2}$, whose elements are square roots of probability densities of a larger (curved) exponential family. In addition, the vectors $\mathscr{P}_{t}\left(\{p(\cdot, \theta)\}^{1 / 2}\right)$ and $Q_{t}^{k}\left(\{p(\cdot, \theta)\}^{1 / 2}\right)$ for $k=0,1, \ldots, d$ are tangent vectors at the point $\{p(\cdot, \theta)\}^{1 / 2}$ to the manifold $\Sigma_{t, \theta}^{1 / 2}$ and the projection can take place within a finite-dimensional tangent space, so that infinite dimensionality is bypassed. The manifolds $\Sigma_{t, \theta}^{1 / 2}$ may be viewed as enveloping manifolds for $\mathrm{EM}^{1 / 2}(c)$. This alternative approach will be used again in Section 8 below.

Let us consider the exponential family $\operatorname{EM}(c)$, as from Definition 2.2, and assume that the coefficients $c$ are differentiable up to order two. From the expression obtained in (14), it follows that

$$
\begin{aligned}
\alpha_{t}(p(\cdot, \theta))= & \frac{\mathscr{B}_{t}^{*} p(\cdot, \theta)}{p(\cdot, \theta)} \\
= & -\sum_{i=1}^{n}\left(f_{t}^{i} \frac{\partial}{\partial x_{i}}\left(\theta^{\mathrm{T}} c\right)+\frac{\partial f_{t}^{i}}{\partial x_{i}}\right) \\
& +\frac{1}{2} \sum_{i, j=1}^{n}\left(a_{t}^{i j} \frac{\partial^{2}}{\partial x_{i} \partial x_{j}}\left(\theta^{\mathrm{T}} c\right)+a_{t}^{i j} \frac{\partial}{\partial x_{i}}\left(\theta^{\mathrm{T}} c\right) \frac{\partial}{\partial x_{j}}\left(\theta^{\mathrm{T}} c\right)+2 \frac{\partial a_{t}^{i j}}{\partial x_{j}} \frac{\partial}{\partial x_{i}}\left(\theta^{\mathrm{T}} c\right)+\frac{\partial^{2} a_{t}^{i j}}{\partial x_{i} \partial x_{j}}\right) .
\end{aligned}
$$


We shall assume that the coefficients $f_{t}, a_{t}$ and $h_{t}$ of the system (7), and the coefficients $c$ of the exponential family $\operatorname{EM}(c)$ satisfy Assumption (D).

Remark 5.1. Sufficient explicit conditions for (D) to hold for $\operatorname{EM}(c)$ can be easily given. For example, (D) will hold if the coefficients $f_{t}$ (and its first derivatives), $a_{t}$ (and its first and second derivatives), $h_{t}, c$ (and its first and second derivatives) have at most polynomial growth, and if densities in $\operatorname{EM}(c)$ integrate any polynomial.

Under Assumption (D) we define below, for any $\theta_{0} \in \Theta$ and any $t_{0} \geqslant 0$, a curved exponential family $\Sigma_{t_{0}, \theta_{0}}$, containing $\operatorname{EM}(c)$. For the definition of a curved exponential family, see Amari (1985, Section 4.2).

Proposition 5.2. Let $\left\{d_{1}, \ldots, d_{s}\right\}$, with $0 \leqslant s \leqslant d+2$, be scalar functions defined on $\mathbf{R}^{n}$ and depending on $t_{0}, \theta_{0}$, such that $\left\{1, c_{1}, \ldots, c_{m}, d_{1}, \ldots, d_{s}\right\}$ is a basis of the linear space

$$
\operatorname{span}\left\{1, c_{1}, \ldots, c_{m}, \alpha_{t_{0}}\left(p\left(\cdot, \theta_{0}\right)\right), \frac{1}{2}\left|h_{t_{0}}\right|^{2}, h_{t_{0}}^{1}, \ldots, h_{t_{0}}^{d}\right\} .
$$

Define

$$
\Sigma_{t_{0}, \theta_{0}}:=\left\{p_{t_{0}, \theta_{0}}(\cdot, \theta, \xi), \theta \in \Theta, \xi \in \Xi\right\}
$$

with

$$
p_{t_{0}, \theta_{0}}(x, \theta, \xi):=\exp \left\{\theta^{\mathrm{T}} c(x)+\xi^{\mathrm{T}} d(x)-\frac{1}{4}|\xi|^{4}|d(x)|^{4}-\psi_{t_{0}, \theta_{0}}(\theta, \xi)\right\},
$$

and where $\Xi \subseteq \mathbf{R}^{s}$ is open.

If Assumption (D) holds, and if $\Xi \subseteq \mathbf{R}^{s}$ is a sufficiently small neighbourhood of the origin, then $\Sigma_{t_{0}, \theta_{0}}^{1 / 2}$ is a $(m+s)$-dimensional submanifold of $L_{2}$.

Remark 5.3. For any $\theta \in \Theta, p\left(\cdot, \theta_{t}\right)=p_{t_{0}, \theta_{0}}(\cdot, \theta, 0)$, and hence $\operatorname{EM}(c) \subset \Sigma_{t_{0}, \theta_{0}}$, which makes $\Sigma_{t_{0}, \theta_{0}}^{1 / 2}$ an enveloping manifold of $\operatorname{EM}^{1 / 2}(c)$.

Proof. For simplicity, we use in this proof the notation $p_{0}(\cdot, \theta, \xi)=p_{t_{0}, \theta_{0}}(\cdot, \theta, \xi)$, and $\psi_{0}(\theta, \xi)=\psi_{t_{0}, \theta_{0}}(\theta, \xi)$. It follows from the Cauchy-Schwartz inequality and the Young inequality $u \leqslant \frac{3}{4}+\frac{1}{4} u^{4}$ that

$$
p_{0}(x, \theta, \xi) \leqslant \exp \left\{\theta^{\mathrm{T}} c(x)+\frac{3}{4}-\psi_{0}(\theta, \xi)\right\} ;
$$

hence $p_{0}(\cdot, \theta, \xi)$ is integrable for any $\theta \in \Theta$, and any $\xi \in \mathbf{R}^{s}$. Define the following expectation parameters:

$$
\begin{aligned}
& \bar{\eta}_{i}(\theta, \xi):=\frac{\partial}{\partial \theta_{i}} \psi_{0}(\theta, \xi)=\mathrm{E}_{p_{0}(\cdot, \theta, \xi)}\left\{c_{i}\right\}, \quad i=1, \ldots, m, \\
& \bar{\chi}_{l}(\theta, \xi):=\frac{\partial}{\partial \xi_{l}} \psi_{0}(\theta, \xi)=\mathrm{E}_{p_{0}(\cdot, \theta, \xi)}\left\{d_{l}-\xi_{l}|\xi|^{2}|d|^{4}\right\}, \quad l=1, \ldots, s,
\end{aligned}
$$

and the associated tangent vectors 


$$
\begin{aligned}
& \frac{\partial}{\partial \theta_{i}}\left\{p_{0}(\cdot, \theta, \xi)\right\}^{1 / 2}=\frac{1}{2}\left\{p_{0}(\cdot, \theta, \xi)\right\}^{1 / 2}\left\{c_{i}-\bar{\eta}_{i}(\theta, \xi)\right\}, \quad i=1, \ldots, m, \\
& \frac{\partial}{\partial \xi_{l}}\left\{p_{0}(\cdot, \theta, \xi)\right\}^{1 / 2}=\frac{1}{2}\left\{p_{0}(\cdot, \theta, \xi)\right\}^{1 / 2}\left\{d_{l}-\xi_{l}|\xi|^{2}|d|^{4}-\bar{\chi}_{l}(\theta, \xi)\right\}, \quad l=1, \ldots, s,
\end{aligned}
$$

at the point $\left\{p_{0}(\cdot, \theta, \xi)\right\}^{1 / 2} \in \Sigma_{t_{0}, \theta_{0}}^{1 / 2}$. Under Assumption (D) we have

$$
\begin{aligned}
\mathrm{E}_{p_{0}(\cdot, \theta, \xi)}\left\{|d|^{2}\right\} & =\mathrm{E}_{p(\cdot, \theta)}\left\{|d|^{2} \exp \left(\xi^{\mathrm{T}} d-\frac{1}{4}|\xi|^{4}|d|^{4}\right)\right\} \exp \left\{\psi(\theta)-\psi_{0}(\theta, \xi)\right\} \\
& \leqslant \mathrm{E}_{p(\cdot, \theta)}\left\{|d|^{2}\right\} \exp \left\{\frac{3}{4}+\psi(\theta)-\psi_{0}(\theta, \xi)\right\} \\
& <\infty
\end{aligned}
$$

and similarly

$$
\begin{aligned}
|\xi|^{6} \mathrm{E}_{p_{0}(\cdot, \theta, \xi)}\left\{|d|^{8}\right\} & =\mathrm{E}_{p(\cdot, \theta)}\left\{|\xi|^{6}|d|^{8} \exp \left(\xi^{\mathrm{T}} d-\frac{1}{4}|\xi|^{4}|d|^{4}\right)\right\} \exp \left\{\psi(\theta)-\psi_{0}(\theta, \xi)\right\} \\
& \leqslant \mathrm{E}_{p(\cdot, \theta)}\left\{|d|^{2}\right\} \max _{u \geqslant 0}\left\{u^{6} \exp \left(u-\frac{1}{4} u^{4}\right)\right\} \exp \left\{\psi(\theta)-\psi_{0}(\theta, \xi)\right\} \\
& <\infty
\end{aligned}
$$

which proves that all the tangent vectors introduced above are in $L_{2}$, and hence the associated Fisher information matrix $\bar{g}(\theta, \xi)$ is well defined.

Finally, it is easy to prove that these tangent vectors are linearly independent, and hence the Fisher information matrix is invertible. Indeed, the following decomposition holds:

$$
|d(x)|^{4}=\alpha+\beta^{\mathrm{T}} c(x)+\gamma^{\mathrm{T}} d(x)+e(x),
$$

where the scalar function $e$ either is zero or is linearly independent of $\left\{1, c_{1}, \ldots, c_{m}\right.$, $\left.d_{1}, \ldots, d_{s}\right\}$, and

$$
\begin{aligned}
0= & \rho+\lambda^{\mathrm{T}}[c-\bar{\eta}(\theta, \xi)]+\mu^{\mathrm{T}}\left[d-\xi|\xi|^{2}|d|^{4}-\bar{\chi}(\theta, \xi)\right] \\
= & \left\{\rho-\lambda^{\mathrm{T}} \bar{\eta}(\theta, \xi)-\mu^{\mathrm{T}} \bar{\chi}(\theta, \xi)-\mu^{\mathrm{T}} \xi|\xi|^{2} \alpha\right\}+\left(\lambda-\mu^{\mathrm{T}} \xi|\xi|^{2} \beta\right)^{\mathrm{T}} c \\
& +\left(\mu-\mu^{\mathrm{T}} \xi|\xi|^{2} \gamma\right)^{\mathrm{T}} d-\mu^{\mathrm{T}} \xi|\xi|^{2} e,
\end{aligned}
$$

implies that

$$
\begin{array}{r}
\rho-\lambda^{\mathrm{T}} \bar{\eta}(\theta, \xi)-\mu^{\mathrm{T}} \bar{\chi}(\theta, \xi)-\mu^{\mathrm{T}} \xi|\xi|^{2} \alpha=0, \\
\lambda-\mu^{\mathrm{T}} \xi|\xi|^{2} \beta=0, \\
\left(I-\gamma \xi \xi^{\mathrm{T}}|\xi|^{2}\right) \mu=\mu-\mu^{\mathrm{T}} \xi|\xi|^{2} \gamma=0 .
\end{array}
$$

If $\xi$ is sufficiently small, the matrix $I-\gamma \xi^{\mathrm{T}}|\xi|^{2}$ is invertible, and hence $\mu=0$, from which we deduce that $\lambda=0$ and $\rho=0$. This establishes the linear independence.

It is easly checked that, for all $\theta \in \Theta$, 
$\operatorname{span}\left\{\frac{1}{2}\{p(\cdot, \theta)\}^{1 / 2} \alpha_{t_{0}}\left(p\left(\cdot, \theta_{0}\right)\right), \frac{1}{2}\{p(\cdot, \theta)\}^{1 / 2} \beta_{t_{0}}^{k}\left(p\left(\cdot, \theta_{0}\right)\right), k=0,1, \ldots, d\right\} \subseteq L_{\{p(\cdot, \theta)\}^{1 / 2}} \Sigma_{t_{0}, \theta_{0}}^{1 / 2}$.

Let us consider (11) in the Stratonovich form for $\left\{p_{t}^{1 / 2}, t \geqslant t_{0}\right\}$, starting at time $t_{0}$ from the initial condition $p_{t_{0}}^{1 / 2}=\left\{p\left(\cdot, \theta_{0}\right)\right\}^{1 / 2} \in \mathrm{EM}^{1 / 2}(c)$ for some $\theta_{0} \in \Theta$, i.e.

$$
\begin{aligned}
\mathrm{d} p_{t}^{1 / 2} & =\frac{1}{2} p_{t}^{1 / 2} \alpha_{t}\left(p_{t}\right) \mathrm{d} t-\frac{1}{2} p_{t}^{1 / 2} \beta_{t}^{0}\left(p_{t}\right) \mathrm{d} t+\frac{1}{2} \sum_{k=1}^{d} p_{t}^{1 / 2} \beta_{t}^{k}\left(p_{t}\right) \circ \mathrm{d} Y_{t}^{k} \\
& =\mathscr{P}_{t}\left(p_{t}^{1 / 2}\right) \mathrm{d} t-\mathcal{Q}_{t}^{0}\left(p_{t}^{1 / 2}\right) \mathrm{d} t+\sum_{k=1}^{d} Q_{t}^{k}\left(p_{t}^{1 / 2}\right) \circ \mathrm{d} Y_{t}^{k}, \quad t \geqslant t_{0} .
\end{aligned}
$$

It is immediate to check that

$$
\mathscr{P}_{t_{0}}\left(p_{t_{0}}^{1 / 2}\right)=\frac{1}{2} p_{t_{0}}^{1 / 2} \alpha_{t_{0}}\left(p_{t_{0}}\right)=\frac{1}{2}\left\{p\left(\cdot, \theta_{0}\right)\right\}^{1 / 2} \alpha_{t_{0}}\left(p\left(\cdot, \theta_{0}\right)\right) \in L_{\left\{p\left(\cdot, \theta_{0}\right)\right\}^{1 / 2}} \Sigma_{t_{0}, \theta_{0}}^{1 / 2}
$$

and

$$
Q_{t_{0}}^{k}\left(p_{t_{0}}^{1 / 2}\right)=\frac{1}{2} p_{t_{0}}^{1 / 2} \beta_{t_{0}}^{k}\left(p_{t_{0}}\right)=\frac{1}{2}\left\{p\left(\cdot, \theta_{0}\right)\right\}^{1 / 2} \beta_{t_{0}}^{k}\left(p\left(\cdot, \theta_{0}\right)\right) \in L_{\left\{p\left(\cdot \theta_{0}\right)\right\}^{1 / 2}} \Sigma_{t_{0}, \theta_{0}}^{1 / 2},
$$

for $k=0,1, \ldots, d$. Then we can project at any time instant $t_{0}$ from the finite-dimensional

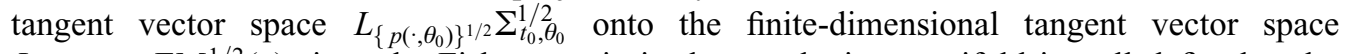
$L_{\left\{p\left(\cdot, \theta_{0}\right)\right\}^{1 / 2}} \mathrm{EM}^{1 / 2}(c)$ since the Fisher metric in the enveloping manifold is well defined under Assumption (D).

Let $\langle\cdot, \cdot\rangle$ be the Fisher information metric on the enveloping manifold at the current point $p\left(\cdot, \theta_{0}\right)=p_{t_{0}, \theta_{0}}\left(\cdot, \theta_{0}, 0\right)$. Consider the orthogonal projection

$$
\begin{aligned}
& \Pi_{t_{0}, \theta_{0}}: L_{\left\{p\left(\cdot, \theta_{0}\right)\right\}^{1 / 2}} \Sigma_{t_{0}, \theta_{0}}^{1 / 2} \rightarrow L_{\left\{p\left(\cdot, \theta_{0}\right)\right\}^{1 / 2}} \operatorname{EM}^{1 / 2}(c), \\
& v \mapsto \sum_{i=1}^{m}\left(\sum_{j=1}^{m} 4 g^{i j}\left(\theta_{0}\right)\left\langle v, \frac{\partial\left\{p\left(\cdot, \theta_{0}\right)\right\}^{1 / 2}}{\partial \theta_{j}}\right\rangle\right) \frac{\partial\left\{p\left(\cdot, \theta_{0}\right)\right\}^{1 / 2}}{\partial \theta_{i}} .
\end{aligned}
$$

The exponential projection filter for the exponential family $\operatorname{EM}(c)$ is defined as the solution of the following SDE on the manifold $\operatorname{EM}^{1 / 2}(c)$ :

$$
\begin{aligned}
\mathrm{d}\left\{p\left(\cdot, \theta_{t}\right)\right\}^{1 / 2}= & \Pi_{t, \theta_{t}} \circ \mathscr{P}_{t}\left(\left\{p\left(\cdot, \theta_{t}\right)\right\}^{1 / 2}\right) \mathrm{d} t-\Pi_{t, \theta_{t}} \circ Q_{t}^{0}\left(\left\{p\left(\cdot, \theta_{t}\right)\right\}^{1 / 2}\right) \mathrm{d} t \\
& +\sum_{k=1}^{d} \Pi_{t, \theta_{t}} \circ Q_{t}^{k}\left(\left\{p\left(\cdot, \theta_{t}\right)\right\}^{1 / 2}\right) \circ \mathrm{d} Y_{t}^{k} .
\end{aligned}
$$

We can now state the main result of this section, which is a consequence of the more general Theorem 4.3 given in Section 4 above.

Theorem 5.4. Assume that the coefficients $f_{t}, a_{t}$ and $h_{t}$ of the system (7), and the coefficients $c$ of the exponential family $\operatorname{EM}(c)$ satisfy (A)-(D).

Then, for all $\theta \in \Theta$ and all $t \geqslant 0$, 
(a) the vectors $\mathscr{P}_{t}\left(\{p(\cdot, \theta)\}^{1 / 2}\right)$ and $Q_{t}^{k}\left(\{p(\cdot, \theta)\}^{1 / 2}\right)$ for $k=0,1, \ldots, d$ are vectors in the tangent space $L_{\{p(; \theta)\}^{1 / 2}} \Sigma_{t, \theta}^{1 / 2}$ of the finite-dimensional time-varying submanifold $\Sigma_{t, \theta}^{1 / 2}$ of $L_{2}$ and

(b) the nonlinear operators $\Pi_{t, \theta} \circ \mathscr{P}_{t}$ and $\Pi_{t, \theta} \circ Q_{t}^{k}$ for $k=0,1, \ldots, d$ are vector fields on the original exponential manifold $\operatorname{EM}^{1 / 2}(c)$.

The projection filter density $p\left(\cdot, \theta_{t}\right)$ is described by (19), and the projection filter parameters satisfy the following $S D E$ :

$$
\begin{aligned}
\mathrm{d} \theta_{t}=\{ & \left.g\left(\theta_{t}\right)\right\}^{-1} \mathrm{E}_{p\left(\cdot, \theta_{t}\right)}\left\{\mathscr{C}_{t} c\right\} \mathrm{d} t-\left\{g\left(\theta_{t}\right)\right\}^{-1} \mathrm{E}_{p\left(\cdot, \theta_{t}\right)}\left\{\frac{1}{2}\left|h_{t}\right|^{2}\left[c-\eta\left(\theta_{t}\right)\right]\right\} \mathrm{d} t \\
& +\left\{g\left(\theta_{t}\right)\right\}^{-1} \sum_{k=1}^{d} \mathrm{E}_{p\left(\cdot, \theta_{t}\right)}\left\{h_{t}^{k}\left[c-\eta\left(\theta_{t}\right)\right]\right\} \circ \mathrm{d} Y_{t}^{k} .
\end{aligned}
$$

Under the assumptions on the coefficients, this equation has a unique solution up to the a.s. positive exit time $\tau:=\inf \left\{t \geqslant 0: \theta_{t} \notin \Theta\right\}$.

Proof. By specializing to the exponential family $\operatorname{EM}(c)$ the general equation (16) for the projection filter parameters, and by using the duality relation

$$
\begin{aligned}
\mathrm{E}_{p\left(\cdot, \theta_{t}\right)}\left\{\frac{\mathscr{L}_{t}^{*} p\left(\cdot, \theta_{t}\right)}{p\left(\cdot, \theta_{t}\right)} \mathrm{D} \log p\left(\cdot, \theta_{t}\right)\right\} & =\int \mathscr{L}_{t}^{*} p\left(x, \theta_{t}\right)\left[c(x)-\eta\left(\theta_{t}\right)\right] \mathrm{d} x \\
& =\int \mathscr{L}_{t} c(x) p\left(x, \theta_{t}\right) \mathrm{d} x \\
& =\mathrm{E}_{p\left(\cdot, \theta_{t}\right)}\left\{\mathscr{L}_{t} c\right\},
\end{aligned}
$$

we obtain

$$
\begin{aligned}
\mathrm{d} \theta_{t}= & \left\{g\left(\theta_{t}\right)\right\}^{-1} \mathrm{E}_{p\left(\cdot, \theta_{t}\right)}\left\{\mathscr{D}_{t} c\right\} \mathrm{d} t-\left\{g\left(\theta_{t}\right)\right\}^{-1} \mathrm{E}_{p\left(\cdot, \theta_{t}\right)}\left\{\frac{1}{2}\left|h_{t}\right|^{2}\left[c-\eta\left(\theta_{t}\right)\right]\right\} \mathrm{d} t \\
& +\left\{g\left(\theta_{t}\right)\right\}^{-1} \sum_{k=1}^{d} \mathrm{E}_{p\left(\cdot, \theta_{t}\right)}\left\{h_{t}^{k}\left[c-\eta\left(\theta_{t}\right)\right]\right\} \circ \mathrm{d} Y_{t}^{k} .
\end{aligned}
$$

Under Assumption (D), the maps

$$
\theta \mapsto \mathrm{E}_{p(\cdot ; \theta)}\left\{\mathscr{C}_{t} c\right\}, \quad \theta \mapsto \mathrm{E}_{p(\cdot, \theta)}\left\{\frac{1}{2}\left|h_{t}(x)\right|^{2}[c-\eta(\theta)]\right\}, \quad \theta \mapsto \mathrm{E}_{p(\cdot, \theta)}\left\{h_{t}^{k}[c-\eta(\theta)]\right\},
$$

for $k=1, \ldots, d$, are locally Lipschitz continuous in $\Theta$, uniformly in $t \geqslant 0$, and we can apply Theorem 4.3 .

Remark 5.5. The initial condition $\theta_{0}$ for (20) is defined as follows: if $p_{0} \in \operatorname{EM}(c)$, then $p_{0}=p\left(\cdot, \theta_{0}\right)$ for some unique $\theta_{0} \in \Theta$, which is used as the initial condition. Otherwise, we project $p_{0}$ on $\operatorname{EM}(c)$, by minimizing the Kullback-Leibler information

$$
K\left(p_{0}, p(\cdot, \theta)\right):=\int \log \left(\frac{p_{0}(x)}{p(x, \theta)}\right) p_{0}(x) \mathrm{d} x,
$$


with respect to $\theta \in \Theta$. After straightforward calculations, and making use of Lemma 2.3, this reduces to maximizing

$$
\left(\theta^{\mathrm{T}} \int c(x) p_{0}(x) \mathrm{d} x-\psi(\theta)\right)
$$

Assuming that the maximum is achieved in $\theta_{0} \in \Theta$, necessary conditions yield

$$
\eta_{i}\left(\theta_{0}\right)=\int c_{i}(x) p_{0}(x) \mathrm{d} x, \quad i=1, \ldots, m
$$

\section{The projection residual and the choice of a convenient exponential family}

In this section, we are interested in defining quantities which will provide estimates of the local error resulting from the projection filter approximation. Compare (11) for the (square root of the) true density $p_{t}$, i.e.

$$
\mathrm{d} p_{t}^{1 / 2}=\mathscr{P}_{t}\left(p_{t}^{1 / 2}\right) \mathrm{d} t-Q_{t}^{0}\left(p_{t}^{1 / 2}\right) \mathrm{d} t+\sum_{k=1}^{d} Q_{t}^{k}\left(p_{t}^{1 / 2}\right) \circ \mathrm{d} Y_{t}^{k},
$$

and (15) for the (square root of the) projection filter density $p_{t}^{\pi}=p\left(\cdot, \theta_{t}\right.$ ), i.e.

$$
\mathrm{d}\left(p_{t}^{\pi}\right)^{1 / 2}=\Pi_{\theta_{t}} \circ \mathscr{P}_{t}\left(\left(p_{t}^{\pi}\right)^{1 / 2}\right) \mathrm{d} t-\Pi_{\theta_{t}} \circ Q_{t}^{0}\left(\left(p_{t}^{\pi}\right)^{1 / 2}\right) \mathrm{d} t+\sum_{k=1}^{d} \Pi_{\theta_{t}} \circ Q_{t}^{k}\left(\left(p_{t}^{\pi}\right)^{1 / 2}\right) \circ \mathrm{d} Y_{t}^{k}
$$

Two steps are involved in using the projection filter density $p\left(\cdot, \theta_{t}\right)$ as an approximation of the true density $p_{t}$. We make a first approximation by evaluating the right-hand side of $(21)$ at the current projection filter density $p\left(\cdot, \theta_{t}\right)$ and not at the true density $p_{t}$. Even with this approximation, the resulting coefficients $\mathscr{P}_{t}\left(\left\{p\left(\cdot, \theta_{t}\right)\right\}^{1 / 2}\right)$ and $\mathscr{Q}_{t}^{k}\left(\left\{p\left(\cdot, \theta_{t}\right)\right\}^{1 / 2}\right)$ for $k=0,1, \ldots, d$ would make the solution leave the manifold $\operatorname{EM}^{1 / 2}(c)$, and we make a

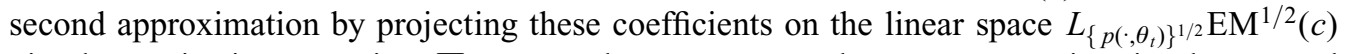
via the projection mapping $\Pi_{\theta_{t}}$. In order to express the error occurring in the second approximation step at time $t$, we define the prediction residual operator $\mathscr{B}_{t}^{\bullet}$ and the correction residual operators $\mathscr{R}_{t}^{k}$ for $k=0,1, \ldots, d$ as follows:

$$
\mathscr{R}_{t}^{\bullet}:=\mathscr{P}_{t}-\Pi_{\theta_{t}} \circ \mathscr{P}_{t} \quad \text { and } \quad \mathscr{R}_{t}^{k}:=Q_{t}^{k}-\Pi_{\theta_{t}} \circ Q_{t}^{k} .
$$

These operators, when applied to the square root of density $\left\{p\left(\cdot, \theta_{t}\right)\right\}^{1 / 2} \in \operatorname{EM}^{1 / 2}(c)$ yield vectors of $L_{2}$. We call such vectors projection residuals; they give a posteriori estimates of the local error resulting from the projection filter approximation. We can compute the norm of such vectors according to the norm $\|\cdot\|$ in $L_{2}$, and we define the prediction residual norm $r_{t}^{\bullet}$ and correction residual norms $r_{t}^{k}$ for $k=0,1, \ldots, d$ as follows:

$$
r_{t}^{\bullet}:=\left\|\mathscr{B}_{t}^{\bullet}\left(\left\{p\left(\cdot, \theta_{t}\right)\right\}^{1 / 2}\right)\right\| \quad \text { and } \quad r_{t}^{k}:=\left\|\mathscr{B}_{t}^{k}\left(\left\{p\left(\cdot, \theta_{t}\right)\right\}^{1 / 2}\right)\right\| .
$$

However, we are still missing a single estimate of the local error resulting from the 
projection. We define below a single residual operator, only in the case where $\mathscr{R}_{t}^{k}=0$ for all $t \geqslant 0$, and all $k=1, \ldots, d$. In this case, we define the total residual operator $\mathscr{B}_{t}$ as

$$
\mathscr{R}_{t}:=\mathscr{R}_{t}^{\bullet}-\mathscr{R}_{t}^{0} \text {, }
$$

and the corresponding total residual norm $r_{t}$ as

$$
r_{t}:=\left\|\mathscr{B}_{t}\left(\left\{p\left(\cdot, \theta_{t}\right)\right\}^{1 / 2}\right)\right\| .
$$

Note that, if in addition $\mathscr{R}_{t}^{0}=0$, then $r_{t}$ reduces to $r_{t}^{\bullet}$. We shall introduce below manifolds $\operatorname{EM}^{1 / 2}\left(c^{\bullet}\right)$ and $\operatorname{EM}^{1 / 2}\left(c^{*}\right)$ for which such a definition is applicable. Now we try to give some intuition for the above definition. Suppose that we replace in (21) and (22) the observation $\left\{Y_{t}, t \geqslant 0\right\}$ with some smooth process $\left\{u_{t}, t \geqslant 0\right\}$, e.g. a regularized approximation, i.e. we consider the equations

$$
\frac{\mathrm{d}}{\mathrm{d} t} p_{t}^{1 / 2}=\mathscr{P}_{t}\left(p_{t}^{1 / 2}\right)-Q_{t}^{0}\left(p_{t}^{1 / 2}\right)+\sum_{k=1}^{d} \mathcal{Q}_{t}^{k}\left(p_{t}^{1 / 2}\right) \dot{u}_{t}^{k}
$$

and

$$
\frac{\mathrm{d}}{\mathrm{d} t}\left(p_{t}^{\pi}\right)^{1 / 2}=\Pi_{\theta_{t}} \circ \mathscr{P}_{t}\left(\left(p_{t}^{\pi}\right)^{1 / 2}\right)-\Pi_{\theta_{t}} \circ \mathscr{Q}_{t}^{0}\left(\left(p_{t}^{\pi}\right)^{1 / 2}\right)+\sum_{k=1}^{d} \Pi_{\theta_{t}} \circ Q_{t}^{k}\left(\left(p_{t}^{\pi}\right)^{1 / 2}\right) \dot{u}_{t}^{k} .
$$

In this case, we can define a single residual operator expressing the difference between the rate of change in the smooth Kushner-Stratonovich equation (23) and the rate of change in the smooth projection filter equation (24), i.e.

$$
\mathscr{R}_{t}^{u}:=\mathscr{R}_{t}^{\bullet}-\mathscr{R}_{t}^{0}+\sum_{k=1}^{d} \mathscr{R}_{t}^{k} \dot{u}_{t}^{k} .
$$

Of course, if we return to the original situation, e.g. letting the regularized approximation $\left\{u_{t}, t \geqslant 0\right\}$ converge to the observation $\left\{Y_{t}, t \geqslant 0\right\}$, there is no limit to the smooth residual operator $\mathscr{B}_{t}^{u}$, unless $\mathscr{B}_{t}^{k}=0$ for all $t \geqslant 0$, and all $k=1, \ldots, d$. In this case only, we define the total residual operator $\mathscr{B}_{t}$ as above.

From now on, and throughout the paper, we assume for simplicity that $h_{t}=h$ does not depend explicitly on time. This is necessary in order to define the simplifying timeinvariant exponential families $\operatorname{EM}\left(c^{\bullet}\right)$ and $\operatorname{EM}\left(c^{*}\right)$ below.

\subsection{The exponential families $\operatorname{EM}\left(c^{\bullet}\right)$ and $\operatorname{EM}\left(c^{*}\right)$}

The exponential family $\operatorname{EM}\left(c^{\bullet}\right)$ is such that the functions $\left\{h^{1}, \ldots, h^{d}, \frac{1}{2}|h|^{2}\right\}$ belong to $\operatorname{span}\left\{1, c_{1}^{\bullet}, \ldots, c_{m}^{\bullet}\right\}$, i.e. such that for all $x \in \mathbf{R}^{n}$

$$
\frac{1}{2}|h(x)|^{2}=\lambda^{0}+\sum_{i=1}^{m} \lambda_{i}^{0} c_{i}^{\bullet}(x), \quad h^{k}(x)=\lambda^{k}+\sum_{i=1}^{m} \lambda_{i}^{k} c_{i}^{\bullet}(x),
$$

for $k=1, \ldots, d$. 
Theorem 6.1. Assume that the coefficients $f_{t}$ and $a_{t}$ of the system (7), and the coefficients $c^{\bullet}$ of the exponential family $\operatorname{EM}\left(c^{\bullet}\right)$ satisfy $(\mathrm{A})-(\mathrm{C})$ and the first assumption in (D).

Then, for the projection filter associated with the exponential family $\operatorname{EM}\left(c^{\bullet}\right)$, the correction residual norms $r_{t}^{k}$ are identically zero for all $t \geqslant 0$, and all $k=0,1, \ldots, d$, and the SDE for the parameters reduces to

$$
\mathrm{d} \theta_{t}=\left\{g\left(\theta_{t}\right)\right\}^{-1} \mathrm{E}_{p\left(\cdot, \theta_{t}\right)}\left\{\mathscr{C}_{t} c^{\bullet}\right\} \mathrm{d} t-\lambda_{\bullet}^{0} \mathrm{~d} t+\sum_{k=1}^{d} \lambda_{\bullet}^{k} \mathrm{~d} Y_{t}^{k},
$$

where for all $k=0,1, \ldots, d$ the $m$-dimensional vector $\lambda_{\bullet}^{k}$ is defined by

$$
\lambda_{\bullet}^{k}=\left[\lambda_{1}^{k} \ldots \lambda_{m}^{k}\right]^{\mathrm{T}} .
$$

Under the assumptions on the coefficients, this equation has a unique solution, up to the a.s. positive exit time $\tau:=\inf \left\{t>0: \theta_{t} \notin \Theta\right\}$.

Proof. All the assumptions of Theorem 5.4 are satisfied, and therefore the solution of the stochastic differential equation for the projection filter with manifold $\operatorname{EM}^{1 / 2}\left(c^{\bullet}\right)$ exists and is unique up to the a.s. positive exit time $\tau$.

Next, we prove that the correction residual norms vanish. Indeed, it follows from (25) that

$$
\begin{aligned}
Q_{t}^{0}\left(\left\{p\left(\cdot, \theta_{t}\right)\right\}^{1 / 2}\right) & =\frac{1}{4}\left[|h|^{2}-\mathrm{E}_{p\left(\cdot, \theta_{t}\right)}\left\{|h|^{2}\right\}\right]\left\{p\left(\cdot, \theta_{t}\right)\right\}^{1 / 2} \\
& =\frac{1}{2} \sum_{i=1}^{m} \lambda_{i}^{0}\left[c_{i}^{\bullet}-\mathrm{E}_{p\left(\cdot, \theta_{t}\right)}\left\{c_{i}^{\bullet}\right\}\right]\left\{p\left(\cdot, \theta_{t}\right)\right\}^{1 / 2}
\end{aligned}
$$

and similarly

$$
\begin{aligned}
Q_{t}^{k}\left(\left\{p\left(\cdot, \theta_{t}\right)\right\}^{1 / 2}\right) & =\frac{1}{2}\left[h^{k}-\mathrm{E}_{p\left(\cdot, \theta_{t}\right)}\left\{h^{k}\right\}\right]\left\{p\left(\cdot, \theta_{t}\right)\right\}^{1 / 2} \\
& =\frac{1}{2} \sum_{i=1}^{m} \lambda_{i}^{k}\left[c_{i}^{\bullet}-\mathrm{E}_{p\left(\cdot, \theta_{t}\right)}\left\{c_{i}^{\bullet}\right\}\right]\left\{p\left(\cdot, \theta_{t}\right)\right\}^{1 / 2},
\end{aligned}
$$

for $k=1, \ldots, d$. We remark that

$$
\frac{1}{2}\left[c_{i}^{\bullet}-\mathrm{E}_{p\left(\cdot, \theta_{t}\right)}\left\{c_{i}^{\bullet}\right\}\right]\left\{p\left(\cdot, \theta_{t}\right)\right\}^{1 / 2}=\frac{1}{2}\left[c_{i}^{\bullet}-\eta_{i}\left(\theta_{t}\right)\right]\left\{p\left(\cdot, \theta_{t}\right)\right\}^{1 / 2}=\frac{\partial\left\{p\left(\cdot, \theta_{t}\right)\right\}^{1 / 2}}{\partial \theta_{i}}
$$

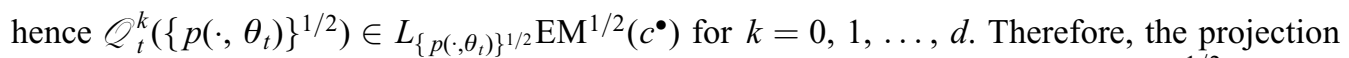
does not modify these vectors since they already lie in the tangent space of $\operatorname{EM}^{1 / 2}\left(c^{\bullet}\right)$.

Finally, the equation for the parameters is obtained via straightforward calculations. Indeed, it follows from (25) that

$$
\mathrm{E}_{p\left(\cdot, \theta_{t}\right)}\left\{\frac{1}{2}|h|^{2}\left[c_{j}^{\bullet}-\eta_{j}\left(\theta_{t}\right)\right]\right\}=\sum_{i^{\prime}=1}^{m} \lambda_{i^{\prime}}^{0} \mathrm{E}_{p\left(\cdot, \theta_{t}\right)}\left\{c_{i^{\prime}}^{\bullet}\left[c_{j}^{\bullet}-\eta_{j}\left(\theta_{t}\right)\right]\right\}=\sum_{i^{\prime}=1}^{m} g_{j i^{\prime}}\left(\theta_{t}\right) \lambda_{i^{\prime}}^{0}
$$

hence 


$$
\sum_{j=1}^{m} g^{i j}\left(\theta_{t}\right) \mathrm{E}_{p\left(\cdot, \theta_{t}\right)}\left\{\frac{1}{2}|h|^{2}\left[c_{j}^{\bullet}-\eta_{j}\left(\theta_{t}\right)\right]\right\}=\sum_{j=1}^{m} g^{i j}\left(\theta_{t}\right) \sum_{i^{\prime}=1}^{m} g_{j i^{\prime}}\left(\theta_{t}\right) \lambda_{i^{\prime}}^{0}=\lambda_{i}^{0},
$$

and similarly

$$
\sum_{j=1}^{m} g^{i j}\left(\theta_{t}\right) \mathrm{E}_{p\left(\cdot, \theta_{t}\right)}\left\{h^{k}\left[c_{j}^{\bullet}-\eta_{j}\left(\theta_{t}\right)\right]\right\}=\lambda_{i}^{k},
$$

for all $k=1, \ldots, d$. Substituting these expressions into the right-hand side of (20) yields (26).

Using Lemma 2.1, we obtain the following expression for the norm of the projection error.

Proposition 6.2. Under the assumptions of Theorem 6.1, the total residual norm $r_{t}=r_{t}^{\bullet}$ satisfies

$$
r_{t}^{2}=\frac{1}{4} \mathrm{E}_{p\left(\cdot, \theta_{t}\right)}\left\{\left|\frac{\mathscr{L}_{t}^{*} p\left(\cdot, \theta_{t}\right)}{p\left(\cdot, \theta_{t}\right)}\right|^{2}\right\}-\frac{1}{4}\left[\mathrm{E}_{p\left(\cdot, \theta_{t}\right)}\left\{\mathscr{D}_{t} c^{\bullet}\right\}\right]^{\mathrm{T}}\left\{g\left(\theta_{t}\right)\right\}^{-1} \mathrm{E}_{p\left(\cdot, \theta_{t}\right)}\left\{\mathscr{L}_{t} c^{\bullet}\right\} .
$$

The diffusion coefficient in the SDE (26) for the parameters is constant, which implies that (26) can be seen as either an Itô or a Stratonovich SDE, so that it satisfies the formal rules of calculus. Moreover, for the numerical solution of such an equation, the simpler Euler scheme coincides with the Milshtein scheme, which is a strongly convergent scheme of order 1 (Kloeden and Platen 1992, Section 10.3).

Note also that we have still some freedom left, and we may wonder whether one can use this to select $m$ and the functions $\left\{c_{1}^{\bullet}, \ldots, c_{m}^{\bullet}\right\}$ in order to reduce the total residual norm $r_{t}$. However, great prudence is needed, because the filter may become complicated and numerical problems may arise. See examples on the cubic sensor in Section 8 of Brigo et al. (1995a). In general, a trade-off is necessary in order to obtain an accurate but still not too involved exponential family and the associated projection filter.

The exponential family $\operatorname{EM}\left(c^{*}\right)$ is such that the functions $\left\{h^{1}, \ldots, h^{d}\right\}$ belong to $\operatorname{span}\left\{1, c_{1}^{*}, \ldots, c_{m}^{*}\right\}$, i.e. such that for all $x \in \mathbf{R}^{n}$

$$
h^{k}(x)=\lambda^{k}+\sum_{i=1}^{m} \lambda_{i}^{k} c_{i}^{*}(x)
$$

for $k=1, \ldots, d$.

Similarly to Theorem 6.1 above, we have the following.

Theorem 6.3. Assume that the coefficients $f_{t}$ and $a_{t}$ of the system (7), and the coefficients $c^{*}$ of the exponential family $\operatorname{EM}\left(c^{*}\right)$ satisfy (A)-(C) and the first assumption in (D).

Then, for the projection filter associated with the exponential family $\operatorname{EM}\left(c^{*}\right)$, the correction residual norms $r_{t}^{k}$ are identically zero for all $t \geqslant 0$, and all $k=1, \ldots, d$, and the $S D E$ for the parameters reduces to 


$$
\mathrm{d} \theta_{t}=\left\{g\left(\theta_{t}\right)\right\}^{-1} \mathrm{E}_{p\left(\cdot, \theta_{t}\right)}\left\{\mathscr{C}_{t} c^{*}\right\} \mathrm{d} t-\left\{g\left(\theta_{t}\right)\right\}^{-1} \mathrm{E}_{p\left(\cdot, \theta_{t}\right)}\left\{\frac{1}{2}|h|^{2}\left[c^{*}-\eta\left(\theta_{t}\right)\right]\right\} \mathrm{d} t+\sum_{k=1}^{d} \lambda_{*}^{k} \mathrm{~d} Y_{t}^{k},
$$

where for all $k=1, \ldots, d$ the m-dimensional vector $\lambda_{*}^{k}$ is defined by

$$
\lambda_{*}^{k}=\left[\lambda_{1}^{k} \ldots \lambda_{m}^{k}\right]^{\mathrm{T}}
$$

Under the assumptions on the coefficients, this equation has a unique solution, up to the a.s. positive exit time $\tau:=\inf \left\{t>0: \theta_{t} \notin \Theta\right\}$.

The proof is analogous to the proof of Theorem 6.1 and is therefore omitted. Using Lemma 2.1, we obtain the following expression for the norm of the projection error.

Proposition 6.4. Under the assumptions of Theorem 6.3, and if the coefficient $|h|^{2}$ is differentiable up to order two, then the total residual norm $r_{t}$ satisfies

$$
\begin{aligned}
r_{t}^{2}= & \frac{1}{4} \mathrm{E}_{p\left(\cdot, \theta_{t}\right)}\left\{\left|\frac{\mathscr{D}_{t}^{*} p\left(\cdot, \theta_{t}\right)}{p\left(\cdot, \theta_{t}\right)}\right|^{2}\right\}-\frac{1}{4} \mathrm{E}_{p\left(\cdot, \theta_{t}\right)}\left\{\mathscr{C}_{t}|h|^{2}\right\}+\frac{1}{16}\left[\mathrm{E}_{p\left(\cdot, \theta_{t}\right)}\left\{|h|^{4}\right\}-\left(\mathrm{E}_{p\left(\cdot, \theta_{t}\right)}\left\{|h|^{2}\right\}\right)^{2}\right] \\
& -\frac{1}{4}\left[\mathrm{E}_{p\left(\cdot, \theta_{t}\right)}\left\{\mathscr{L}_{t} c^{*}-\frac{1}{2}|h|^{2}\left[c^{*}-\eta\left(\theta_{t}\right)\right]\right\}\right]^{\mathrm{T}}\left\{g\left(\theta_{t}\right)\right\}^{-1} \mathrm{E}_{p\left(\cdot, \theta_{t}\right)}\left\{\mathscr{B}_{t} c^{*}-\frac{1}{2}|h|^{2}\left[c^{*}-\eta\left(\theta_{t}\right)\right]\right\} .
\end{aligned}
$$

Proof. Using the definitions (12) and (13), (5) yields

$$
\begin{aligned}
r_{t}^{2}= & \frac{1}{4} \mathrm{E}_{p\left(\cdot, \theta_{t}\right)}\left\{\left|\alpha_{t}\left(p\left(\cdot, \theta_{t}\right)\right)-\beta_{t}^{0}\left(p\left(\cdot, \theta_{t}\right)\right)\right|^{2}\right\} \\
& -\frac{1}{4}\left[\mathrm{E}_{p\left(\cdot, \theta_{t}\right)}\left\{\mathscr{C}_{t} c^{*}-\frac{1}{2}|h|^{2}\left[c^{*}-\eta\left(\theta_{t}\right)\right]\right\}\right]^{\mathrm{T}}\left\{g\left(\theta_{t}\right)\right\}^{-1} \mathrm{E}_{p\left(\cdot, \theta_{t}\right)}\left\{\mathscr{B}_{t} c^{*}-\frac{1}{2}|h|^{2}\left[c^{*}-\eta\left(\theta_{t}\right)\right]\right\} .
\end{aligned}
$$

Obviously

$$
\mathrm{E}_{p\left(\cdot, \theta_{t}\right)}\left\{\left|\beta_{t}^{0}\left(p\left(\cdot, \theta_{t}\right)\right)\right|^{2}\right\}=\frac{1}{4}\left[\mathrm{E}_{p\left(\cdot, \theta_{t}\right)}\left\{|h|^{4}\right\}-\left(\mathrm{E}_{p\left(\cdot, \theta_{t}\right)}\left\{|h|^{2}\right\}\right)^{2}\right]
$$

and, if the coefficient $|h|^{2}$ is differentiable up to order two, the following duality relation holds:

$$
\begin{aligned}
\mathrm{E}_{p\left(\cdot, \theta_{t}\right)}\left\{\alpha_{t}\left(p\left(\cdot, \theta_{t}\right)\right) \beta_{t}^{0}\left(p\left(\cdot, \theta_{t}\right)\right)\right\} & =\int \mathscr{L}_{t}^{*} p\left(x, \theta_{t}\right) \frac{1}{2}\left[|h(x)|^{2}-\mathrm{E}_{p\left(\cdot, \theta_{t}\right)}\left\{|h|^{2}\right\}\right] \mathrm{d} x \\
& =\frac{1}{2} \int \mathscr{L}_{t}|h|^{2}(x) p\left(x, \theta_{t}\right) \mathrm{d} x \\
& =\frac{1}{2} \mathrm{E}_{p\left(\cdot, \theta_{t}\right)}\left\{\mathscr{C}_{t}|h|^{2}\right\} .
\end{aligned}
$$




\subsection{The case of discrete-time observations}

Additional evidence for the choice of the exponential family $\operatorname{EM}\left(c^{\bullet}\right)$ is obtained by considering the case of a nonlinear filtering problem with discrete-time observations. In this model, the state process is as in (7), i.e.

$$
\mathrm{d} X_{t}=f_{t}\left(X_{t}\right) \mathrm{d} t+\sigma_{t}\left(X_{t}\right) \mathrm{d} W_{t}, \quad X_{0},
$$

but only discrete-time observations are available,

$$
z_{n}=h\left(X_{t_{n}}\right)+v_{n},
$$

at times $0=t_{0}<t_{1}<\cdots<t_{n}<\cdots$, where $\left\{v_{n}, n \geqslant 0\right\}$ is a Gaussian white-noise sequence with unit variance and independent of $\left\{X_{t}, t \geqslant 0\right\}$.

The nonlinear filtering problem consists in finding the conditional density $p_{n}(x)$ of the state $X_{t_{n}}$ given the observations up to time $t_{n}$, i.e. such that $\mathbf{P}\left[X_{t_{n}} \in \mathrm{d} x \mid \mathscr{Z}_{n}\right]=p_{n}(x) \mathrm{d} x$, where $\mathscr{Z}_{n}:=\sigma\left(z_{0}, \ldots, z_{n}\right)$. We define also the prediction conditional density $p_{n}^{-}(x) \mathrm{d} x=\mathbf{P}\left[X_{t_{n}} \in \mathrm{d} x \mid \mathscr{E}_{n-1}\right]$. The sequence $\left\{p_{n}, n \geqslant 0\right\}$ satisfies a recurrence equation, and the transition from $p_{n-1}$ to $p_{n}$ is decomposed in two steps, as explained by Jazwinski (1970, Theorem 6.1).

Prediction step. Between time $t_{n-1}$ and $t_{n}$, we solve the Fokker-Planck equation

$$
\frac{\partial p_{t}^{n}}{\partial t}=\mathscr{B}_{t}^{*} p_{t}^{n}, \quad p_{t_{n-1}}^{n}=p_{n-1} .
$$

The solution at final time $t_{n}$ defines the prediction conditional density $p_{n}^{-}=p_{t_{n}}^{n}$.

Correction step. At time $t_{n}$, the observation $z_{n}$ is combined with the prediction conditional density $p_{n}^{-}$via the Bayes rule

$$
p_{n}(x)=c_{n} \Psi_{n}(x) p_{n}^{-}(x),
$$

where $c_{n}$ is a normalizing constant, and $\Psi_{n}(x)$ denotes the likelihood function for the estimation of $X_{t_{n}}$ based on the observation $z_{n}$ only, i.e.

$$
\Psi_{n}(x):=\exp \left\{-\frac{1}{2}\left|z_{n}-h(x)\right|^{2}\right\} .
$$

If we use the exponential family $\operatorname{EM}\left(c^{\bullet}\right)$ defined above, then we obtain the projection filter density $p\left(\cdot, \theta_{n}\right)$, and the transition from $\theta_{n-1}$ to $\theta_{n}$ is also decomposed in two steps.

Prediction step. Between time $t_{n-1}$ and $t_{n}$, we solve the ordinary differential equation

$$
\dot{\theta}_{t}^{n}=\left\{g\left(\theta_{t}^{n}\right)\right\}^{-1} \mathrm{E}_{p\left(\cdot, \theta_{t}^{n}\right)}\left\{\mathscr{L}_{t} c^{\bullet}\right\}, \quad \theta_{t_{n-1}}^{n}=\theta_{n-1} .
$$

The solution at final time $t_{n}$ defines the prediction parameters $\theta_{n}^{-}=\theta_{t_{n}}^{n}$.

Correction step. Substituting the approximation $p\left(\cdot, \theta_{n}^{-}\right)$into $(30)$, we observe that the 
resulting density does not leave the exponential family $\operatorname{EM}\left(c^{\bullet}\right)$. Indeed, it follows from (25) and (31) that

$$
\begin{aligned}
\Psi_{n}(x) & =\exp \left(-\frac{1}{2}|h(x)|^{2}+\sum_{k=1}^{d} h^{k}(x) z_{n}^{k}-\frac{1}{2}\left|z_{n}\right|^{2}\right) \\
& =\exp \left\{-\lambda^{0}-\sum_{i=1}^{m} \lambda_{i}^{0} c_{i}^{\bullet}(x)+\sum_{k=1}^{d} \lambda^{k} z_{n}^{k}+\sum_{i=1}^{m}\left(\sum_{k=1}^{d} \lambda_{i}^{k} z_{n}^{k}\right) c_{i}^{\bullet}(x)-\frac{1}{2}\left|z_{n}\right|^{2}\right\},
\end{aligned}
$$

and the parameters are updated according to the formula

$$
\theta_{n}=\theta_{n}^{-}-\lambda_{\bullet}^{0}+\sum_{k=1}^{d} \lambda_{\bullet}^{k} z_{n}^{k},
$$

which is exact.

\section{Assumed density filters}

Because the equations of nonlinear filtering are generally intractable, many approximation methods have been proposed. A well-known approximation method is the EKF, in which the conditional first- and second-order moments are approximated by using a linearization procedure. A potential disadvantage of such a method is that no use is made of the general nonlinear filtering equations; after linearization the formulae for linear Gaussian filtering are applied. If one tries to develop approximation schemes starting from the nonlinear filtering equations, one is confronted with the problem that the conditional densities (if they exist) do not belong in general to any finite-dimensional class of densities. One heuristic way to deal with this problem is to consider the moment equations and to assume arbitrarily that the conditional densities belong to some finite-dimensional class of densities, even if this is known to be wrong. The resulting moment equations will in general be inconsistent but, by selecting carefully a limited number of moment equations, one can obtain a consistent definition of an approximate filter, which is called an assumed density filter in the literature (Kushner 1967; Maybeck 1979, Section 12.7).

As will be shown, it also matters whether the selected moment equations are taken in the Itô or in the Stratonovich form. In order to discuss such assumed density filters properly, and to study their relation with the projection filters in Section 8 below, we give now a more formal definition of assumed density filters.

Throughout the remaining part of the paper we assume that, in addition to Assumptions (A)-(C) of Section 3, the coefficient $f_{t}$ of the system (7) has at most polynomial growth when $|x|$ goes to infinity. (Note that, under Assumption (B), the coefficient $a_{t}$ has at most quadratic growth.) Consider any twice differentiable function $c$ which, together with its derivatives up to order two, has at most polynomial growth when $|x|$ goes to infinity. Then the conditions given by Fujisaki et al. (1972, Theorem 4.1) are fulfilled for the $c$ moments to satisfy (8), i.e. 


$$
\mathrm{d} \pi_{t}(c)=\pi_{t}\left(\mathscr{L}_{t} c\right) \mathrm{d} t+\sum_{k=1}^{d}\left\{\pi_{t}\left(h_{t}^{k} c\right)-\pi_{t}\left(h_{t}^{k}\right) \pi_{t}(c)\right\} \mathrm{d} Y_{t}^{k}
$$

The Stratonovich version of this equation is obtained directly from (9), i.e.

$$
\begin{aligned}
\mathrm{d} \pi_{t}(c)= & \pi_{t}\left(\mathscr{C}_{t} c\right) \mathrm{d} t-\frac{1}{2}\left\{\pi_{t}\left(\left|h_{t}\right|^{2} c\right)-\pi_{t}\left(\left|h_{t}\right|^{2}\right) \pi_{t}(c)\right\} \mathrm{d} t \\
& +\sum_{k=1}^{d}\left\{\pi_{t}\left(h_{t}^{k} c\right)-\pi_{t}\left(h_{t}^{k}\right) \pi_{t}(c)\right\} \circ \mathrm{d} Y_{t}^{k}
\end{aligned}
$$

and holds under the conditions just described.

The following is a generalization of the concept of assumed conditional probability density filters as introduced in Kushner (1967).

Definition 7.1. Consider a finite set $\left\{c_{1}, \ldots, c_{m}\right\}$ of twice differentiable scalar functions defined on $\mathbf{R}^{n}$, such that each $c_{i}, i=1, \ldots, m$ and its derivatives up to order two have at most polynomial growth. Consider a corresponding m-dimensional family $\{\pi(\cdot, \eta)$, $\left.\eta=\left(\eta_{1}, \ldots, \eta_{m}\right) \in \mathscr{E}\right\}$ of probability measures, where $\mathscr{E} \subset \mathbf{R}^{m}$ is open, such that each element of the family satisfies the equations

$$
\eta_{i}=\mathrm{E}_{\eta}\left\{c_{i}\right\}, \quad i=1, \ldots, m,
$$

and is uniquely specified by these equations. Here $\mathrm{E}_{\eta}\{\cdot\}$ denotes the expectation with respect to the probability measure $\pi(\cdot, \eta)$.

In accordance with the ADF principle, the Itô-based ADF is defined by the Itô SDEs

$$
\mathrm{d} \eta_{t}^{i}=\mathrm{E}_{\eta_{t}}\left\{\mathscr{L}_{t} c_{i}\right\} \mathrm{d} t+\sum_{k=1}^{d}\left(\mathrm{E}_{\eta_{t}}\left\{h_{t}^{k} c_{i}\right\}-\mathrm{E}_{\eta_{t}}\left\{h_{t}^{k}\right\} \eta_{t}^{i}\right)\left(\mathrm{d} Y_{t}^{k}-\mathrm{E}_{\eta_{t}}\left\{h_{t}^{k}\right\} \mathrm{d} t\right), \quad i=1, \ldots, m
$$

Similarly the Stratonovich-based ADF is defined by the Stratonovich SDEs

$$
\begin{aligned}
\mathrm{d} \eta_{t}^{i}= & \mathrm{E}_{\eta_{t}}\left\{\mathscr{B}_{t} c_{i}\right\} \mathrm{d} t-\frac{1}{2}\left(\mathrm{E}_{\eta_{t}}\left\{\left|h_{t}\right|^{2} c_{i}\right\}-\mathrm{E}_{\eta_{t}}\left\{\left|h_{t}\right|^{2}\right\} \eta_{t}^{i}\right) \mathrm{d} t \\
& +\sum_{k=1}^{d}\left(\mathrm{E}_{\eta_{t}}\left\{h_{t}^{k} c_{i}\right\}-\mathrm{E}_{\eta_{t}}\left\{h_{t}^{k}\right\} \eta_{t}^{i}\right) \circ \mathrm{d} Y_{t}^{k}, \quad i=1, \ldots, m .
\end{aligned}
$$

Note that in the following we shall work with exponential families such as $\operatorname{EM}(c)$. However, the class of probability measures that satisfies the moment conditions for $c$ uniquely, in the above definition, is larger than the class of measures whose densities are in $\operatorname{EM}(c)$.

Although this may be surprising at first, the Itô-based ADF and the Stratonovich-based $\mathrm{ADF}$ are different filters in general. This will be shown by working out the Itô-based and Stratonovich-based Gaussian assumed density filters for the cubic sensor problem. The fact that they are different is due to the inconsistency that is inherent to the ADF principle; applying this principle to equivalent representations of the same equation leads to different results. 
Example 7.2 (Stratonovich-based Gaussian ADF for the cubic sensor). Consider the scalar system

$$
\begin{aligned}
& \mathrm{d} X_{t}=\sigma \mathrm{d} W_{t}, \quad X_{0}, \\
& \mathrm{~d} Y_{t}=X_{t}^{3} \mathrm{~d} t+\mathrm{d} V_{t}, \quad Y_{0}=0,
\end{aligned}
$$

where the initial state $X_{0}$ and the standard Brownian motions $\left\{W_{t}, t \geqslant 0\right\}$ and $\left\{V_{t}, t \geqslant 0\right\}$ are mutually independent, and where $\sigma$ is a real constant. Let us compute the Stratonovich-based ADF for this system using a Gaussian family, i.e. choosing $c_{1}(x)=x$, and $c_{2}(x)=x^{2}$. Then one obtains $\mu=\eta_{1}=\mathrm{E}_{\eta}\{x\}$, and $\eta_{2}=\mathrm{E}_{\eta}\left\{x^{2}\right\}$, which indeed parametrize the Gaussian family over R. Define $P:=\mathrm{E}_{\eta}\left\{(x-\mu)^{2}\right\}=\eta_{2}-\eta_{1}^{2}$. In the Gaussian case, one has the following relations between the centred higher-order moments up to order eight, and the variance $P$ :

$$
\begin{gathered}
\mathrm{E}_{\eta}\{x-\mu\}=\mathrm{E}_{\eta}\left\{(x-\mu)^{3}\right\}=\mathrm{E}_{\eta}\left\{(x-\mu)^{5}\right\}=\mathrm{E}_{\eta}\left\{(x-\mu)^{7}\right\}=0, \\
\mathrm{E}_{\eta}\left\{(x-\mu)^{4}\right\}=3 P^{2}, \quad \mathrm{E}_{\eta}\left\{(x-\mu)^{6}\right\}=15 P^{3}, \quad \mathrm{E}_{\eta}\left\{(x-\mu)^{8}\right\}=105 P^{4} .
\end{gathered}
$$

Making use of (35), (33) results in the following Stratonovich-based Gaussian ADF:

$$
\begin{aligned}
& \mathrm{d} \mu_{t}=\left(-3 \mu_{t}^{5} P_{t}-30 \mu_{t}^{3} P_{t}^{2}-45 \mu_{t} P_{t}^{3}\right) \mathrm{d} t+\left(3 \mu_{t}^{2} P_{t}+3 P_{t}^{2}\right) \circ \mathrm{d} Y_{t}, \\
& \mathrm{~d} P_{t}=\left(\sigma^{2}-15 \mu_{t}^{4} P_{t}^{2}-90 \mu_{t}^{2} P_{t}^{3}-45 P_{t}^{4}\right) \mathrm{d} t+6 \mu_{t} P_{t}^{2} \circ \mathrm{d} Y_{t} .
\end{aligned}
$$

This should be compared with the Itô-based ADF for the same problem, with the same family of probability densities and the same choice of functions $c_{1}$ and $c_{2}$.

Example 7.3 (Itô-based Gaussian ADF for the cubic sensor). Making use of (35), (32) results in the following Itô-based Gaussian ADF:

$$
\begin{aligned}
& \mathrm{d} \mu_{t}=\left(-3 \mu_{t}^{5} P_{t}-12 \mu_{t}^{3} P_{t}^{2}-9 \mu_{t} P_{t}^{3}\right) \mathrm{d} t+\left(3 \mu_{t}^{2} P_{t}+3 P_{t}^{2}\right) \mathrm{d} Y_{t}, \\
& \mathrm{~d} P_{t}=\left(\sigma^{2}-15 \mu_{t}^{4} P_{t}^{2}-36 \mu_{t}^{2} P_{t}^{3}-9 P_{t}^{4}\right) \mathrm{d} t+6 \mu_{t} P_{t}^{2} \mathrm{~d} Y_{t} .
\end{aligned}
$$

Putting these Itô equations in the Stratonovich form one obtains the Stratonovich version of the Itô-based ADF:

$$
\begin{aligned}
& \mathrm{d} \mu_{t}=\left(-3 \mu_{t}^{5} P_{t}-30 \mu_{t}^{3} P_{t}^{2}-36 \mu_{t} P_{t}^{3}\right) \mathrm{d} t+\left(3 \mu_{t}^{2} P_{t}+3 P_{t}^{2}\right) \circ \mathrm{d} Y_{t}, \\
& \mathrm{~d} P_{t}=\left(\sigma^{2}-15 \mu_{t}^{4} P_{t}^{2}-81 \mu_{t}^{2} P_{t}^{3}-18 P_{t}^{4}\right) \mathrm{d} t+6 \mu_{t} P_{t}^{2} \circ \mathrm{d} Y_{t} .
\end{aligned}
$$

By comparing the Stratonovich-based Gaussian ADF given in (36) with the Stratonovich version of the Itô-based Gaussian ADF given in (37), we see that these two filters are different.

As is clear from the definition, the construction of an ADF depends on the choice of a stochastic calculus, either the Itô or the Stratonovich calculus, and involves both the choice of functions $\left\{c_{1}, \ldots, c_{m}\right\}$ and the choice of an $m$-dimensional family of probability 
distributions which are characterized uniquely by the vector $\eta=\left(\eta_{1}, \ldots, \eta_{m}\right)$, where $\eta_{i}=\mathrm{E}_{\eta}\left\{c_{i}\right\}$ for $i=1, \ldots, m$. Suppose that one wants to work with a specific set of functions $\left\{c_{1}, \ldots, c_{m}\right\}$. Then one way to obtain a family of densities which has the desired property is by using the concept of maximum entropy; given the functions $\left\{c_{1}, \ldots, c_{m}\right\}$ and the vector $\eta=\left(\eta_{1}, \ldots, \eta_{m}\right)$, the probability density $p$ with maximal entropy under the conditions $\mathrm{E}_{p}\left\{c_{i}\right\}=\eta_{i}$ for all $i=1, \ldots, m$, belongs to the exponential family $\operatorname{EM}(c)$, provided that the vector $\eta$ is such that there exists at least one probability density satisfying the conditions (Kagan et al. 1973, Theorem 13.2.1). In the next section it will be shown that, if such an exponential family is chosen, then the Stratonovich-based ADF can be interpreted as a projection filter. The projection filter can be safely defined only via the Stratonovich calculus, as remarked at the beginning of Section 4, and therefore does not lead to the inconsistency aspects which partly afflict the ADFs.

\section{Equivalence between assumed density filters and the projection filter}

The main theorem of this second part of the paper can now be stated. We shall present a proof of this theorem based on stochastic calculus, and we shall also outline a second possible proof which has been carried out in detail by Brigo et al. (1996b, Section 6), or by Brigo (1996a, Section 5.3). The first proof is more elegant and concise, but it does not give much insight into the geometric nature of the result. The second proof relies more on geometric concepts. It uses explicitly projections on the tangent spaces and is based on a crucial result from the theory of information geometry, i.e. the biorthogonality relations between the tangent vectors associated with the canonical parameters, and the tangent vectors associated with the expectation parameters (Amari 1985, Section 2.3). This relationship extends partly from the selected exponential manifold $\mathrm{EM}^{1 / 2}(c)$ to its enveloping manifold; see (38) below. This fact is fundamental in the second proof and further motivates the introduction of the enveloping manifold.

Theorem 8.1. For any exponential family $\operatorname{EM}(c)$, the projection filter $p\left(\cdot, \theta_{t}\right)$ defined by $(20)$ coincides with the Stratonovich-based assumed density filter $p_{E}\left(\cdot, \eta_{t}\right)$ defined by (33).

Proof. We start from (20) for the projection filter canonical parameters, i.e.

$$
\begin{aligned}
\mathrm{d} \theta_{t}=\{ & \left.g\left(\theta_{t}\right)\right\}^{-1} \mathrm{E}_{p\left(\cdot, \theta_{t}\right)}\left\{\mathscr{C}_{t} c\right\} \mathrm{d} t-\left\{g\left(\theta_{t}\right)\right\}^{-1} \mathrm{E}_{p\left(\cdot, \theta_{t}\right)}\left\{\frac{1}{2}\left|h_{t}\right|^{2}\left[c-\eta\left(\theta_{t}\right)\right]\right\} \mathrm{d} t \\
& +\left\{g\left(\theta_{t}\right)\right\}^{-1} \sum_{k=1}^{d} \mathrm{E}_{p\left(\cdot, \theta_{t}\right)}\left\{h_{t}^{k}\left[c-\eta\left(\theta_{t}\right)\right]\right\} \circ \mathrm{d} Y_{t}^{k} .
\end{aligned}
$$

According to Remark 2.4, the expectation parameters can be expressed in terms of the canonical parameters as

$$
\eta_{i}=\eta_{i}(\theta)=\mathrm{E}_{p(\cdot, \theta)}\left\{c_{i}\right\}=\mathrm{E}_{p_{E}(\cdot, \eta)}\left\{c_{i}\right\},
$$


with derivatives

$$
\frac{\partial \eta_{i}}{\partial \theta_{j}}(\theta)=g_{i j}(\theta)
$$

The chain rule for the Stratonovich integrals immediately gives

$$
\begin{aligned}
\mathrm{d} \eta_{t}= & g\left(\theta_{t}\right) \circ \mathrm{d} \theta_{t} \\
= & \mathrm{E}_{p_{E}\left(\cdot, \eta_{t}\right)}\left\{\mathscr{C}_{t} c\right\} \mathrm{d} t-\mathrm{E}_{p_{E}\left(\cdot, \eta_{t}\right)}\left\{\frac{1}{2}\left|h_{t}\right|^{2}\left[c-\eta_{t}\right]\right\} \mathrm{d} t \\
& +\sum_{k=1}^{d} \mathrm{E}_{p_{E}\left(\cdot, \eta_{t}\right)}\left\{h_{t}^{k}\left[c-\eta_{t}\right]\right\} \circ \mathrm{d} Y_{t}^{k},
\end{aligned}
$$

which is exactly (33) obtained using the assumed density filter idea.

Now we outline the key steps of the second proof of Theorem 8.1. First, we fix $t_{0} \geqslant 0$ and $\theta_{0} \in \Theta$, and for simplicity we use the notation $p_{0}(\cdot, \theta, \xi)=p_{t_{0}, \theta_{0}}(\cdot, \theta, \xi)$, and $\psi_{0}(\theta, \xi)=\psi_{t_{0}, \theta_{0}}(\theta, \xi)$. We recall that the expectation parameters for the enveloping manifold $\Sigma_{t_{0}, \theta_{0}}^{1 / 2}$ are defined by (18) and it can be shown (Brigo 1996a, Theorem 5.3.2; Brigo et al. 1996b, Theorem 6.2) that the expectation parameters $\left(\bar{\eta}_{1}, \ldots, \bar{\eta}_{m}, \bar{\chi}_{1}, \ldots, \bar{\chi}_{s}\right)$ provide indeed another (local) parametrization of the enveloping manifold. It is then possible to define tangent vectors associated with the expectation parameters, together with the tangent vectors associated with the canonical parameters:

$$
\begin{aligned}
\partial_{i}(\theta, \xi):=\frac{\partial}{\partial \theta_{i}}\left\{p_{0}(\cdot, \theta, \xi)\right\}^{1 / 2}, & \partial^{i}(\theta, \xi):=\frac{\partial}{\partial \bar{\eta}_{i}}\left\{p_{0}(\cdot, \theta, \xi)\right\}^{1 / 2}, \quad i=1, \ldots, m, \\
\partial_{m+l}(\theta, \xi):=\frac{\partial}{\partial \xi_{l}}\left\{p_{0}(\cdot, \theta, \xi)\right\}^{1 / 2}, & \partial^{m+l}(\theta, \xi):=\frac{\partial}{\partial \bar{\chi}_{l}}\left\{p_{0}(\cdot, \theta, \xi)\right\}^{1 / 2}, \quad l=1, \ldots, s,
\end{aligned}
$$

at point $\left\{p_{0}(\cdot, \theta, \xi)\right\}^{1 / 2} \in \Sigma_{t_{0}, \theta_{0}}^{1 / 2}$. Accordingly, we shall adopt the following notation for vectors tangent to $\mathrm{EM}^{1 / 2}(c)$ :

$$
\partial_{i}(\theta):=\frac{\partial}{\partial \theta_{i}}\{p(\cdot, \theta)\}^{1 / 2}, \quad \partial^{i}(\theta):=\frac{\partial}{\partial \eta_{i}}\{p(\cdot, \theta)\}^{1 / 2}, \quad i=1, \ldots, m .
$$

Let us consider (11) in the Stratonovich form for $\left\{p_{t}^{1 / 2}, t \geqslant t_{0}\right\}$, starting at time $t_{0}$ from the initial condition $p_{t_{0}}^{1 / 2}=\left\{p_{E}\left(\cdot, \eta_{0}\right)\right\}^{1 / 2} \in \mathrm{EM}^{1 / 2}(c)$ with $\eta_{0}=\eta\left(\theta_{0}\right) \in \mathscr{E}$. If we decompose the tangent vectors of $\Sigma_{t_{0}, \theta_{0}}^{1 / 2}$ appearing on the right-hand side of this equation at time $t_{0}$ on the basis associated with the expectation parameters, we obtain

$$
\begin{aligned}
& \mathscr{P}_{t_{0}}\left(\left\{p_{E}\left(\cdot, \eta_{0}\right)\right\}^{1 / 2}\right)=\sum_{i=1}^{m} p_{i}\left(\eta_{0}\right) \partial^{i}\left(\theta_{0}, 0\right)+\sum_{l=1}^{s} p_{m+l}\left(\eta_{0}\right) \partial^{m+l}\left(\theta_{0}, 0\right), \\
& \mathcal{Q}_{t_{0}}^{k}\left(\left\{p_{E}\left(\cdot, \eta_{0}\right)\right\}^{1 / 2}\right)=\sum_{i=1}^{m} q_{i}^{k}\left(\eta_{0}\right) \partial^{i}\left(\theta_{0}, 0\right)+\sum_{l=1}^{s} q_{m+l}^{k}\left(\eta_{0}\right) \partial^{m+l}\left(\theta_{0}, 0\right),
\end{aligned}
$$


for $k=0,1, \ldots, d$. A first fundamental result (Brigo 1996a, Theorem 5.3; Brigo et al. 1996b, Theorem 6.2) is that the biorthogonality relationship (6) for $\operatorname{EM}^{1 / 2}(c)$ partly extends to the enveloping manifold, in the sense that

$$
\begin{aligned}
\left\langle\partial_{j}(\theta, \xi), \partial^{i}(\theta, \xi)\right\rangle & =\frac{1}{4} \delta_{i, j}, \quad i=1, \ldots, m, \\
\left\langle\partial_{j}(\theta, \xi), \partial^{m+l}(\theta, \xi)\right\rangle & =0, \quad l=1, \ldots, s,
\end{aligned}
$$

for all $j=1, \ldots, m$. Secondly, it is easily checked that for all $\theta \in \Theta$

$$
\partial_{j}(\theta)=\partial_{j}(\theta, 0)
$$

for all $j=1, \ldots, m$. It follows from (38) that

$$
\begin{aligned}
p_{i}\left(\eta_{0}\right) & =4\left\langle\mathscr{P}_{t_{0}}\left(\left\{p_{E}\left(\cdot, \eta_{0}\right)\right\}^{1 / 2}\right), \partial_{i}\left(\theta_{0}\right)\right\rangle \\
& =4\left\langle\frac{1}{2}\left\{p_{E}\left(\cdot, \eta_{0}\right)\right\}^{1 / 2} \alpha_{t_{0}}\left(p_{E}\left(\cdot, \eta_{0}\right)\right), \frac{1}{2}\left\{p_{E}\left(\cdot, \eta_{0}\right)\right\}^{1 / 2}\left[c_{i}-\eta_{0}^{i}\right]\right\rangle \\
& =\mathrm{E}_{p_{E}\left(\cdot, \eta_{0}\right)}\left\{\alpha_{t_{0}}\left(p_{E}\left(\cdot, \eta_{0}\right)\right)\left[c_{i}-\eta_{0}^{i}\right]\right\},
\end{aligned}
$$

and similarly

$$
q_{i}^{k}\left(\eta_{0}\right)=\mathrm{E}_{p_{E}\left(\cdot, \eta_{0}\right)}\left\{\beta_{t_{0}}^{k}\left(p_{E}\left(\cdot, \eta_{0}\right)\right)\left[c_{i}-\eta_{0}^{i}\right]\right\},
$$

for $k=0,1, \ldots, d$. It was also proved by Brigo (1996a, Theorem 5.3) and Brigo et al. (1996b, Theorem 6.2) that projecting on $\operatorname{EM}^{1 / 2}(c)$ tangent vectors of $\Sigma_{t_{0}, \theta_{0}}^{1 / 2}$ which are decomposed on the basis associated with the expectation parameters $\left(\bar{\eta}_{1}, \ldots, \bar{\eta}_{m}, \bar{\chi}_{1}, \ldots, \bar{\chi}_{s}\right)$ simply results in eliminating the components associated with the expectation parameters $\left(\bar{\chi}_{1}, \ldots, \bar{\chi}_{s}\right)$. This property is also based on the extension result (38) and yields

$$
\begin{aligned}
& \Pi_{t_{0}, \theta_{0}} \circ \mathscr{P}_{t_{0}}\left(\left\{p_{E}\left(\cdot, \eta_{0}\right)\right\}^{1 / 2}\right)=\sum_{i=1}^{m} p_{i}\left(\eta_{0}\right) \partial^{i}\left(\theta_{0}\right), \\
& \Pi_{t_{0}, \theta_{0}} \circ \mathscr{Q}_{t_{0}}^{k}\left(\left\{p_{E}\left(\cdot, \eta_{0}\right)\right\}^{1 / 2}\right)=\sum_{i=1}^{m} q_{i}^{k}\left(\eta_{0}\right) \partial^{i}\left(\theta_{0}\right),
\end{aligned}
$$

for $k=0,1, \ldots, k$. Since $t_{0} \geqslant 0$ and $\theta_{0} \in \Theta$ are arbitrary, the projection filter for the exponential family $\operatorname{EM}(c)$ is given by the equation

$$
\mathrm{d}\left\{p_{E}\left(\cdot, \eta_{t}\right)\right\}^{1 / 2}=\sum_{i=1}^{m}\left(p_{i}\left(\eta_{t}\right) \mathrm{d} t-q_{i}^{0}\left(\eta_{t}\right) \mathrm{d} t+\sum_{k=1}^{d} q_{i}^{k}\left(\eta_{t}\right) \circ \mathrm{d} Y_{t}^{k}\right) \partial^{i}\left(\theta_{t}\right) .
$$

By expanding $\left\{p_{E}\left(\cdot, \eta_{t}\right)\right\}^{1 / 2}$ according to the Stratonovich chain rule

$$
\mathrm{d}\left\{p_{E}\left(\cdot, \eta_{t}\right)\right\}^{1 / 2}=\sum_{i=1}^{m} \partial^{i}\left(\theta_{t}\right) \circ \mathrm{d} \eta_{t}^{i}
$$


and comparing with (39) we obtain the following SDE for the expectation parameters:

$$
\mathrm{d} \eta_{t}^{i}=p_{i}\left(\eta_{t}\right) \mathrm{d} t-q_{i}^{0}\left(\eta_{t}\right) \mathrm{d} t+\sum_{k=1}^{d} q_{i}^{k}\left(\eta_{t}\right) \circ \mathrm{d} Y_{t}^{k}, \quad i=1, \ldots, m,
$$

which is (33) for the Stratonovich-based ADF associated with $\operatorname{EM}(c)$. This ends the outline of the geometric proof.

The equivalence between the Stratonovich-based ADF and the projection filter is shown to hold for exponential families. In general, for other families of distributions such equivalence does not hold. This can be seen from the following simple example in which we consider a particular curved (Gaussian) exponential family.

Example 8.2 (Projection filter with a curved Gaussian family). Consider the scalar system

$$
\begin{gathered}
\mathrm{d} X_{t}=f\left(X_{t}\right) \mathrm{d} t+\sigma\left(X_{t}\right) \mathrm{d} W_{t}, \quad X_{0}, \\
\mathrm{~d} Y_{t}=X_{t} \mathrm{~d} t+\mathrm{d} V_{t}, \quad Y_{0}=0,
\end{gathered}
$$

where the coefficients $f$ and $a:=\sigma \sigma^{\mathrm{T}}$ satisfy Assumptions (A) and (B), and where the initial state $X_{0}$ and the standard Brownian motions $\left\{W_{t}, t \geqslant 0\right\}$ and $\left\{V_{t}, t \geqslant 0\right\}$ are mutually independent. Choose the following curved family of Gaussian densities:

$$
S:=\{p(\cdot, \theta), \theta \in \mathbf{R} \backslash\{0\}\}, \quad p(x, \theta):=\exp \left\{\theta x-\theta^{2} x^{2}-\psi(\theta)\right\},
$$

where $p(\cdot, \theta)$ is the Gaussian density with mean $1 / 2 \theta$ and variance $1 / 2 \theta^{2}$. We shall denote by $\mathrm{E}_{\theta}\{\cdot\}$ the expectation with respect to the density $p(\cdot, \theta)$. Note that $\eta=\mathrm{E}_{\theta}\{x\}=1 / 2 \theta$. The densities in the above curved Gaussian family may be characterized by $\eta$ as well. We denote by $\mathrm{E}_{\eta}\{\cdot\}$ the corresponding expectation. Note that, since $\eta=1 / 2 \theta$, the Stratonovich chain rule yields $\mathrm{d} \eta_{t}=-1 / 2 \theta_{t}^{2} \circ \mathrm{d} \theta_{t}$. Then, the general equation (16) for the projection filter results in

$$
\mathrm{d} \eta_{t}=-\frac{1}{5}\left(\mathrm{E}_{\eta_{t}}\{f\}-\frac{2}{\eta_{t}} \mathrm{E}_{\eta_{t}}\{x f\}-\frac{2}{\eta_{t}} \mathrm{E}_{\eta_{t}}\{\sigma\}+6 \eta_{t}^{3}\right) \mathrm{d} t+\frac{2}{5} \eta_{t}^{2} \circ \mathrm{d} Y_{t} .
$$

On the other hand, (33) yields instead

$$
\mathrm{d} \eta_{t}=\left(\mathrm{E}_{\eta_{t}}\{f\}-\frac{5}{2} \eta_{t}^{3}\right) \mathrm{d} t+2 \eta_{t}^{2} \circ \mathrm{d} Y_{t},
$$

making use of (35).

One of the striking features of Theorem 8.1 is that it yields a characterization of the projection filters for exponential families in terms of assumed density filters, which are not intrinsically based on differential geometry and can be understood without using geometric concepts.

Finally we observe that as the Itô-based ADF and the Stratonovich-based ADF are different, the theorems proved above state that for a general exponential family $\operatorname{EM}(c)$ the 
equivalence with the projection filter holds only for the Stratonovich-based ADF. However, it can be shown that the Stratonovich-based ADF and the Itô-based ADF coincide for special choices of the exponential family, such as the family $\operatorname{EM}\left(c^{*}\right)$ introduced in $\operatorname{Section}$ 6 , which is constructed in such a way that the functions $\left\{h^{1}, \ldots, h^{k}\right\}$ belong to $\operatorname{span}\left\{1, c_{1}^{*}, \ldots, c_{m}^{*}\right\}$. This provides more evidence for the choice of the exponential family $\operatorname{EM}\left(c^{*}\right)$ (which contains the exponential family $\operatorname{EM}\left(c^{\bullet}\right)$ as a particular case).

Theorem 8.3. For the exponential family $\operatorname{EM}\left(c^{*}\right)$, the Itô-based assumed density filter coincides with the Stratonovich-based assumed density filter, i.e. the solutions of (32) and (33) coincide.

Proof. It follows from (27) that

$$
\frac{1}{2}|h|^{2}=\frac{1}{2} \sum_{k=1}^{d}\left|h^{k}\right|^{2}=\frac{1}{2} \sum_{k=1}^{d}\left|\lambda^{k}\right|^{2}+\sum_{k=1}^{d} \sum_{j=1}^{m} \lambda^{k} \lambda_{j}^{k} c_{j}^{*}+\frac{1}{2} \sum_{k=1}^{d} \sum_{j, j^{\prime}=1}^{m} \lambda_{j}^{k} \lambda_{j^{\prime}}^{k} c_{j}^{*} c_{j^{\prime}}^{*} .
$$

By specializing to the exponential family $\operatorname{EM}\left(c^{*}\right)$ the general equation (33) for the Stratonovich-based ADF, and using Lemma 2.3, we obtain

$$
\begin{aligned}
\mathrm{d} \eta_{t}^{i}= & \mathrm{E}_{p_{E}\left(\cdot, \eta_{t}\right)}\left\{\mathscr{C}_{t} c_{i}^{*}\right\} \mathrm{d} t-\sum_{k=1}^{d} \sum_{j=1}^{m} \lambda^{k} \lambda_{j}^{k}\left[\mathrm{E}_{p_{E}\left(\cdot, \eta_{t}\right)}\left\{c_{j}^{*} c_{i}^{*}\right\}-\mathrm{E}_{p_{E}\left(\cdot, \eta_{t}\right)}\left\{c_{j}^{*}\right\} \eta_{t}^{i}\right] \mathrm{d} t \\
& -\frac{1}{2} \sum_{k=1}^{d} \sum_{j, j^{\prime}=1}^{m} \lambda_{j}^{k} \lambda_{j^{\prime}}^{k}\left[\mathrm{E}_{p_{E}\left(\cdot, \eta_{t}\right)}\left\{c_{j}^{*} c_{j^{\prime}}^{*} c_{i}^{*}\right\}-\mathrm{E}_{p_{E}\left(\cdot, \eta_{t}\right)}\left\{c_{j}^{*} c_{j^{\prime}}^{*}\right\} \eta_{t}^{i}\right] \mathrm{d} t \\
& +\sum_{k=1}^{d} \sum_{j=1}^{m} \lambda_{j}^{k}\left[\mathrm{E}_{p_{E}\left(\cdot, \eta_{t}\right)}\left\{c_{j}^{*} c_{i}^{*}\right\}-\mathrm{E}_{p_{E}\left(\cdot, \eta_{t}\right)}\left\{c_{j}^{*}\right\} \eta_{t}^{i}\right] \circ \mathrm{d} Y_{t}^{k} \\
= & \mathrm{E}_{p_{E}\left(\cdot, \eta_{t}\right)}\left\{\mathscr{C}_{t} c_{i}^{*}\right\} \mathrm{d} t-\sum_{k=1}^{d} \sum_{j=1}^{m} g_{i j}\left(\eta_{t}\right) \lambda_{j}^{k} \lambda^{k} \mathrm{~d} t-\sum_{k=1}^{d} \sum_{j, j^{\prime}=1}^{m} g_{i j}\left(\eta_{t}\right) \lambda_{j}^{k} \lambda_{j^{\prime}}^{k} \eta_{t}^{j^{\prime}} \mathrm{d} t \\
& -\frac{1}{2} \sum_{k=1}^{d} \sum_{j, j^{\prime}=1}^{m} \frac{\partial g_{i j}}{\partial \theta_{j^{\prime}}}\left(\eta_{t}\right) \lambda_{j}^{k} \lambda_{j^{\prime}}^{k} \mathrm{~d} t+\sum_{k=1}^{d} \sum_{j=1}^{m} g_{i j}\left(\eta_{t}\right) \lambda_{j}^{k} \circ \mathrm{d} Y_{t}^{k},
\end{aligned}
$$

for $i=1, \ldots, m$. It is easily checked that the Itô-Stratonovich transformation yields

$$
g_{i j}\left(\eta_{t}\right) \mathrm{d} Y_{t}^{k}=g_{i j}\left(\eta_{t}\right) \circ \mathrm{d} Y_{t}^{k}-\frac{1}{2} \sum_{j^{\prime}=1}^{m} \frac{\partial g_{i j}}{\partial \theta_{j^{\prime}}}\left(\eta_{t}\right) \lambda_{j^{\prime}}^{k} \mathrm{~d} t,
$$

for all $k=1, \ldots, d$ and all $i=1, \ldots, m$. On the other hand, by specializing to the exponential family $\operatorname{EM}\left(c^{*}\right)$ the general equation (32) for the Itô-based ADF, and using Lemma 2.3, we obtain directly 


$$
\begin{aligned}
\mathrm{d} \eta_{t}^{i}= & \mathrm{E}_{p_{E}\left(\cdot, \eta_{t}\right)}\left\{\mathscr{C}_{t} c_{i}^{*}\right\} \mathrm{d} t+\sum_{k=1}^{d} \sum_{j=1}^{m} \lambda_{j}^{k}\left[\mathrm{E}_{p_{E}\left(\cdot \eta_{t}\right)}\left\{c_{j}^{*} c_{i}^{*}\right\}-\mathrm{E}_{p_{E}\left(\cdot, \eta_{t}\right)}\left\{c_{j}^{*}\right\} \eta_{t}^{i}\right] \\
& \times\left(\mathrm{d} Y_{t}^{k}-\lambda^{k} \mathrm{~d} t-\sum_{j^{\prime}=1}^{m} \lambda_{j^{\prime}}^{k} \mathrm{E}_{p_{E}\left(\cdot, \eta_{t}\right)}\left\{c_{j^{\prime}}^{*}\right\} \mathrm{d} t\right) \\
= & \mathrm{E}_{p_{E}\left(\cdot, \eta_{t}\right)}\left\{\mathscr{C}_{t} c_{i}^{*}\right\} \mathrm{d} t-\sum_{k=1}^{d} \sum_{j=1}^{m} g_{i j}\left(\eta_{t}\right) \lambda_{j}^{k} \lambda^{k} \mathrm{~d} t-\sum_{k=1}^{d} \sum_{j, j^{\prime}=1}^{m} g_{i j}\left(\eta_{t}\right) \lambda_{j}^{k} \lambda_{j^{\prime}}^{k} \eta_{t}^{j^{\prime}} \mathrm{d} t \\
& +\sum_{k=1}^{d} \sum_{j=1}^{m} g_{i j}\left(\eta_{t}\right) \lambda_{j}^{k} \mathrm{~d} Y_{t}^{k},
\end{aligned}
$$

for $i=1, \ldots, m$.

\section{Numerical simulations for the cubic sensor}

In this section, we apply the exponential projection filter to the cubic sensor model (34), and we present some simulation results. This system is interesting for several reasons. The state equation is very simple, and yet the optimal filter for the cubic sensor is infinite dimensional, as proved by Hazewinkel et al. (1983), which ensures that we are really facing a problem of approximating an infinite-dimensional filter by a finite-dimensional filter.

The chosen exponential manifold is $\operatorname{EM}^{1 / 2}\left(x, x^{2}, x^{3}, x^{4}\right)$ which is associated with an exponential family with fourth-degree polynomials in the exponent. Since $h(x)=x^{3}$, we can apply Theorem 6.3. The equation of this projection filter and the numerical scheme which was used to implement it have been presented in detail by Brigo et al. (1995a, Sections 8.2 and 9) and Brigo (1996a, Sections 4.6.2 and 4.7).

Equation (28) reduces to

$$
\mathrm{d} \theta_{t}=\left\{g\left(\theta_{t}\right)\right\}^{-1}\left\{\gamma\left(\theta_{t}\right)-\gamma^{0}\left(\theta_{t}\right)\right\} \mathrm{d} t+\lambda \mathrm{d} Y_{t},
$$

where $\lambda=\left[\begin{array}{llll}0 & 0 & 1 & 0\end{array}\right]^{\mathrm{T}}$, and where for all $\theta \in \Theta$

$$
\begin{aligned}
\gamma(\theta):=\mathrm{E}_{p(\cdot, \theta)}\{\mathscr{b} c\}=\sigma^{2}\left[\begin{array}{c}
0 \\
1 \\
3 \eta_{1}(\theta) \\
6 \eta_{2}(\theta)
\end{array}\right], \\
\gamma^{0}(\theta):=\mathrm{E}_{p(\cdot, \theta)}\left\{\frac{1}{2}|h|^{2}[c-\eta(\theta)]\right\}=\frac{1}{2}\left[\begin{array}{c}
\eta_{7}(\theta)-\eta_{6}(\theta) \eta_{1}(\theta) \\
\eta_{8}(\theta)-\eta_{6}(\theta) \eta_{2}(\theta) \\
\eta_{9}(\theta)-\eta_{6}(\theta) \eta_{3}(\theta) \\
\eta_{10}(\theta)-\eta_{6}(\theta) \eta_{4}(\theta)
\end{array}\right],
\end{aligned}
$$

and by definition 


$$
\eta_{i}(\theta):=\mathrm{E}_{p(\cdot, \theta)}\left\{x^{i}\right\}
$$

for any integer $i$.

Using (29), the square of the total residual norm $r_{t}$ is given by

$$
r_{t}^{2}=\frac{1}{4} r_{11}\left(\theta_{t}\right)-\frac{1}{4} r_{12}\left(\theta_{t}\right)+\frac{1}{16} r_{22}\left(\theta_{t}\right)-\frac{1}{4}\left\{\gamma\left(\theta_{t}\right)-\gamma^{0}\left(\theta_{t}\right)\right\}^{\mathrm{T}}\left\{g\left(\theta_{t}\right)\right\}^{-1}\left\{\gamma\left(\theta_{t}\right)-\gamma^{0}\left(\theta_{t}\right)\right\},
$$

where for all $\theta \in \Theta$

$$
\begin{gathered}
r_{12}(\theta):=\mathrm{E}_{p(\cdot, \theta)}\left\{\mathscr{L}|h|^{2}\right\}=15 \sigma^{2} \eta_{4}(\theta), \\
r_{22}(\theta):=\mathrm{E}_{p(\cdot, \theta)}\left\{|h|^{4}-\left(\mathrm{E}_{p(\cdot, \theta)}\left\{|h|^{2}\right\}\right)^{2}\right\}=\eta_{12}(\theta)-\eta_{6}^{2}(\theta),
\end{gathered}
$$

and (after long but straightforward calculations)

$$
\begin{aligned}
r_{11}(\theta):= & \mathrm{E}_{p(\cdot, \theta)}\left\{\left|\frac{\mathscr{B}^{*} p(\cdot, \theta)}{p(\cdot, \theta)}\right|^{2}\right\} \\
= & \sigma^{4}\left\{6 \theta_{4}+2 \theta_{2}^{2}+3 \theta_{1} \theta_{3}+\left(18 \theta_{2} \theta_{3}+12 \theta_{1} \theta_{4}\right) \eta_{1}(\theta)\right. \\
& \left.+\left(48 \theta_{2} \theta_{4}+27 \theta_{3}^{2}\right) \eta_{2}(\theta)+120 \theta_{3} \theta_{4} \eta_{3}(\theta)+120 \theta_{4}^{2} \eta_{4}(\theta)\right\} .
\end{aligned}
$$

To compute efficiently the quantities $\eta_{1}(\theta), \ldots, \eta_{12}(\theta)$, the following key property has been used in our implementation.

Lemma 9.1. In the special case $\operatorname{EM}\left(x, x^{2}, \ldots, x^{m}\right)$ where $n=1$ and the coefficients are monomials in the variable $x$, the entries of the Fisher information matrix satisfy

$$
g_{i j}(\theta)=\eta_{i+j}(\theta)-\eta_{i}(\theta) \eta_{j}(\theta),
$$

and the following identity holds:

$$
\theta_{1} \eta_{i+1}(\theta)+2 \theta_{2} \eta_{i+2}(\theta)+\cdots+m \theta_{m} \eta_{i+m}(\theta)= \begin{cases}0, & \text { if } i=-1, \\ (i+1) \eta_{i}(\theta), & \text { if } i=0,1, \ldots\end{cases}
$$

Equation (40) has been proved by Brigo (1996a, Lemma 3.3.3) and allows one to compute recursively all the moments from the $m-2$ first moments $\eta_{1}(\theta), \ldots, \eta_{m-2}(\theta)$. As a result, the main steps of our algorithm are as follows.

(i) For $i=0,1,2$, compute

$$
I_{i}(\theta)=\int_{-\infty}^{\infty} x^{i} \exp \left\{\theta_{1} x+\theta_{2} x^{2}+\theta_{3} x^{3}+\theta_{4} x^{4}\right\} \mathrm{d} x \quad \text { and } \quad \eta_{i}(\theta)=\frac{I_{i}(\theta)}{I_{0}(\theta)} .
$$

(ii) Compute recursively the higher-order moments $\eta_{3}(\theta), \ldots, \eta_{12}(\theta)$ using (40), i.e.

$$
\eta_{3}(\theta)=-\frac{1}{4 \theta_{4}}\left\{\theta_{1}+2 \theta_{2} \eta_{1}(\theta)+3 \theta_{3} \eta_{2}(\theta)\right\}
$$

and

$$
\eta_{i+4}(\theta)=-\frac{1}{4 \theta_{4}}\left\{(i+1) \eta_{i}(\theta)+\theta_{1} \eta_{i+1}(\theta)+2 \theta_{2} \eta_{i+2}(\theta)+3 \theta_{3} \eta_{i+3}(\theta)\right\}
$$


for $i=0, \ldots, 8$.

(iii) Compute the square of the total residual norm

$$
r^{2}=\frac{1}{4} r_{11}(\theta)-\frac{1}{4} r_{12}(\theta)+\frac{1}{16} r_{22}(\theta)-\frac{1}{4}\left\{\gamma(\theta)-\gamma^{0}(\theta)\right\}^{\mathrm{T}}\{g(\theta)\}^{-1}\left\{\gamma(\theta)-\gamma^{0}(\theta)\right\} .
$$

(iv) Update the parameter $\theta$ using the Euler scheme

$$
\theta \leftarrow \theta+\{g(\theta)\}^{-1}\left\{\gamma(\theta)-\gamma^{0}(\theta)\right\} \Delta t+\lambda \Delta Y,
$$

and go to step (i).

Once a numerical approximation of the projection filter parameters $\theta_{t}$ has been computed, we can compare the corresponding density $p_{t}^{\pi}=p\left(\cdot, \theta_{t}\right)$ to the solution $p_{t}$ of the KushnerStratonovich equation, i.e. to the optimal filter density. Actually, a numerical approximation of $p_{t}$ was used, based on a discretization of the state space with approximately 400 grid points, and on numerical techniques for the solution of stochastic partial differential equations (see, for example, Cai et al. (1995)). The comparison between numerical approximations of the densities $p_{t}^{\pi}$ and $p_{t}$ can be done qualitatively, based on graphical outputs, or we can compute (a numerical approximation of) some distance, such as the Kullback-Leibler information $K\left(p_{t}, p_{t}^{\pi}\right)$ or the Hellinger distance $H\left(p_{t}, p_{t}^{\pi}\right)$. We can also compute an approximation of the total residual norm $r_{t}$ which depends only on the projection filter density.

The simulation results show that the projection filter density is usually very close to the optimal filter density, when the latter is not too sharp (i.e. not too close to a Dirac mass). What would be missing in a Gaussian assumed density filter or in an EKF is the possibility to

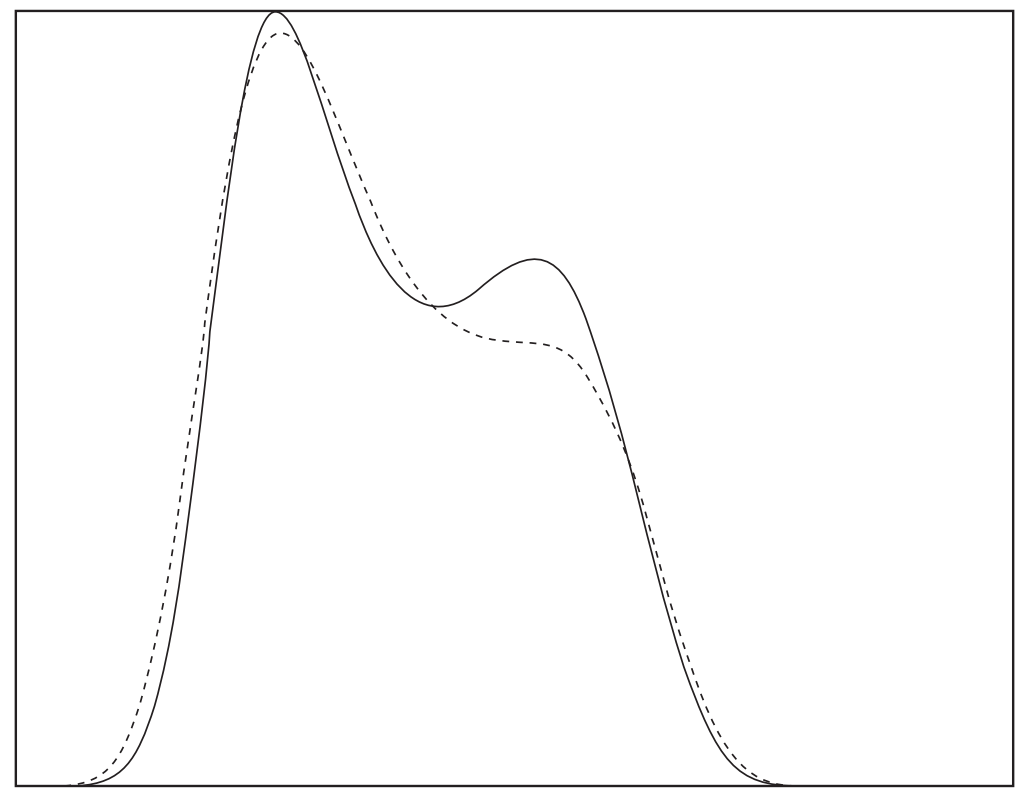

Figure 1. Optimal filter density (-) and projection filter density (-- - ) at time 4.12. 
allow bimodality in the filter density. As the fourth-degree exponential family allows such bimodality, in principle the optimal filter density could be approximated at least qualitatively by a density in this family. This was actually observed in our simulations (Figure 1).

Moreover, we can have an a posteriori indication of the accuracy of the projection filter approximation from the graphical representation of the total residual norm as a function of time. Indeed, there are time instants where the optimal filter and the projection filter first moments are different, but these are exactly the time instants where the total residual norm exhibits large values (Figures 2 and 3). An additional observation that we could make on our simulations is that after a reasonably small time the total residual norm returns towards zero, and correspondingly the projection filter density is again very close to the optimal filter density.

Further details on the simulation results have been given by Brigo et al. (1995a).

\section{Conclusion, and directions of further research}

In this paper we have introduced a new and systematic way of designing approximate finitedimensional filters.

One major issue left is the choice of the exponential family $\operatorname{EM}(c)$. A first answer has been given in Section 6, but this does not completely solve the problem; with the choice of

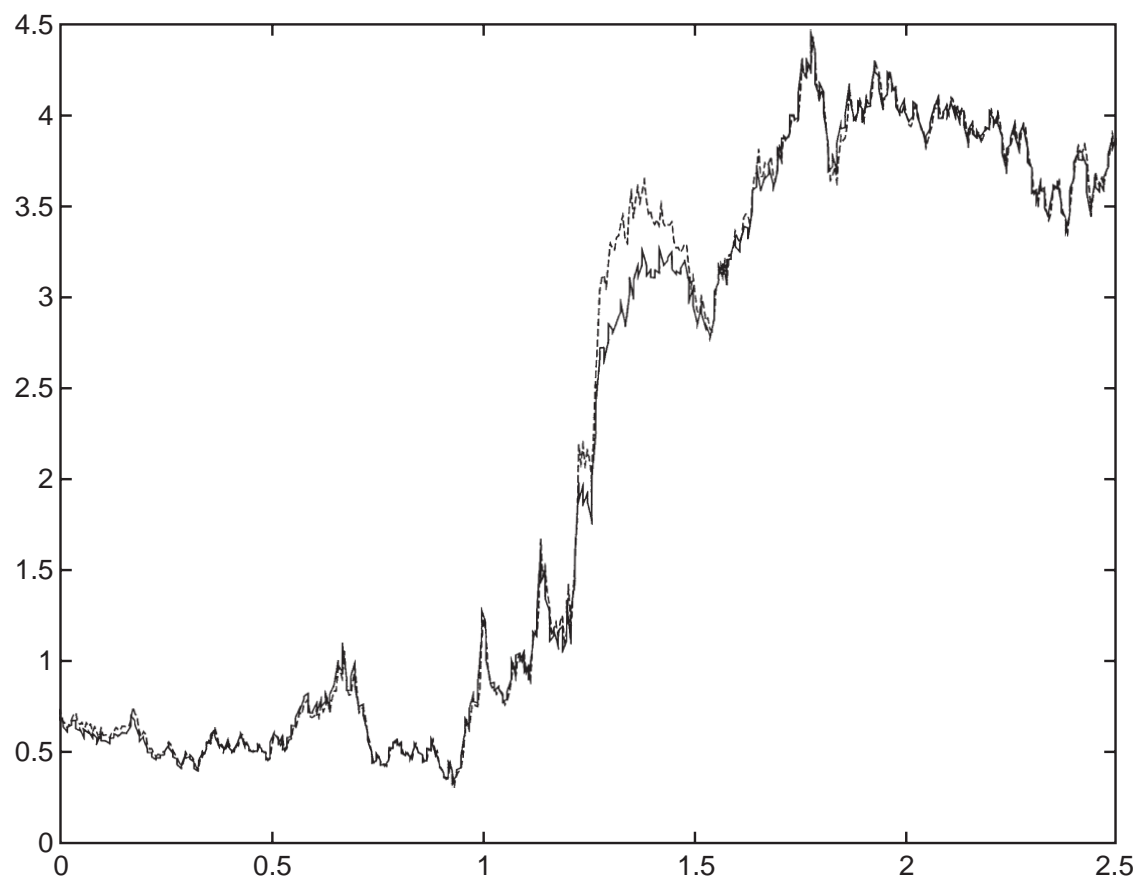

Figure 2. Optimal filter mean value $(-)$ and projection filter mean value $(----)$ between time 0 and 2.5 . 


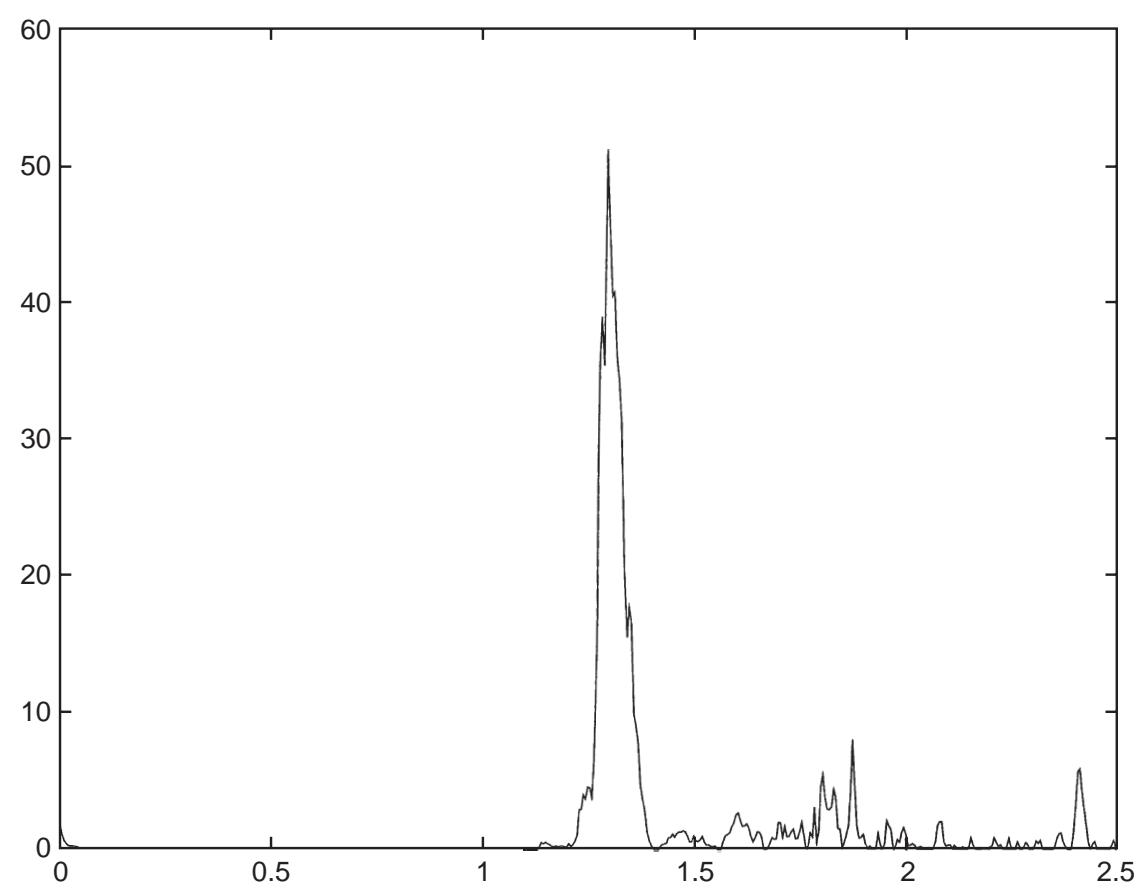

Figure 3. Total residual norm $r_{t}^{*}(-)$ between time 0 and 2.5 .

the family $\operatorname{EM}\left(c^{\bullet}\right)$ there is still some freedom left in the choice of the dimension $m$ and in the choice of the functions $\left\{c_{1}^{\bullet}, \ldots, c_{m}^{\bullet}\right\}$, which could be used to reduce the total residual norm $r_{t}$.

This freedom could also be used to design an adaptive scheme for the choice of the exponential family $\operatorname{EM}(c)$. In this respect, it would also be useful to obtain for all $t \geqslant 0$ an estimate of the distance between the optimal filter density $p_{t}$ and the projection filter density $p\left(\cdot, \theta_{t}\right)$, in terms of the total residual norm history $\left\{r_{s}, 0 \leqslant s \leqslant t\right\}$.

Finally, we would like to define projection filters for discrete-time systems and relate this problem to the work of Kulhavý $(1990 ; 1992 ; 1996)$. Another motivation for this further study will be to obtain efficient numerical schemes for the solution of the SDE satisfied by the projection filter parameters, i.e. (20) for a general family $\operatorname{EM}(c)$, or (26) for the family $\operatorname{EM}\left(c^{\bullet}\right)$.

Each of these problems requires further investigation and will be addressed in subsequent work.

\section{Acknowledgements}

This work was partially supported by the Commission of the European Communities, under the SCIENCE project System Identification, project SC1*-CT92-0779, and under the HCM 
project Statistical Inference for Stochastic Processes, project CHRX-CT92-0078, and by the Army Research Office, under grant DAAH04-95-1-0164. Damiano Brigo was also supported by an INDAM F. Severi Senior Fellowship, and by a TMR grant from the Commission of the European Communities, grant FMBI-CT96-0791.

\section{References}

Amari, S. (1985) Differential-Geometrical Methods in Statistics. Lecture Notes Statist., 28. Berlin: Springer-Verlag.

Barndorff-Nielsen, O. (1978) Information and Exponential Families. New York: Wiley.

Brigo, D. (1995) On the nice behaviour of the Gaussian projection filter with small observation noise. Syst. Control Lett., 26, 363-370.

Brigo, D. (1996a) Filtering by projection on the manifold of exponential densities. PhD Thesis, Department of Economics and Econometrics, Vrije Universiteit, Amsterdam.

Brigo, D. (1996b) New results on the Gaussian projection filter with small observation noise. Syst. Control Lett., 28, 273-279.

Brigo, D. (1997) On nonlinear SDE's whose densities evolve in a finite-dimensional family. In I. Csiszàr and Gy. Michaletzky (eds), Stochastic Differential and Difference Equations, Györ 1996, pp. 11-19. Progression Systems and Control Theory 23. Boston: Birkhäuser.

Brigo, D. and Pistone, G. (1996) Projecting the Fokker-Planck equation onto a finite dimensional exponential family. Preprint 4, Departimento di Matematica, Università di Padova.

Brigo, D., Hanzon, B. and Le Gland, F. (1995a) A differential geometric approach to nonlinear filtering: the projection filter. Publication Interne 914, IRISA.

Brigo, D., Hanzon, B. and Le Gland, F. (1995b) A differential geometric approach to nonlinear filtering: the projection filter. Proceedings of the 34th Conference on Decision and Control, New Orleans 1995, pp. 4006-4011. New York: IEEE.

Brigo, D., Hanzon, B. and Le Gland, F. (1996a) The exponential projection filter and the selection of the exponential family. Proceedings of the Second Portuguese Conference on Automatic Control, Porto 1996, pp. 251-256. APCA.

Brigo, D., Hanzon, B. and Le Gland, F. (1996b) On the relationship between assumed density filters and projection filters. Discussion Paper TI 7-96-18, Tinbergen Institute.

Brigo, D., Hanzon, B. and Le Gland, F. (1998) A differential geometric approach to nonlinear filtering: the projection filter. IEEE Trans Automatic Control, 43, 247-252.

Cai, Z., Le Gland, F. and Zhang, H. (1995) An adaptive local grid refinement method for nonlinear filtering. Publication Interne 954, IRISA.

Davis, M. and Marcus, S. (1981) An introduction to nonlinear filtering. In M. Hazewinkel and J. Willems (eds), Stochastic Systems: the Mathematics of Filtering and Identification and Applications, Les Arcs. 1980. NATO Adv. Stud. Ser. D, C78. Dordrecht: Reidel.

Elworthy, K. (1982) Stochastic Differential Equations on Manifolds. London Math. Soc. Lecture Note Ser., 70. Cambridge: Cambridge University Press.

Fujisaki, M., Kallianpur, G. and Kunita, H. (1972) Stochastic differential equations for the non-linear filtering problem. Osaka J. Math., 9, 19-40.

Hanzon, B. (1987) A differential-geometric approach to approximate nonlinear filtering. In C. Dodson (ed.), Geometrization of Statistical Theory, pp. 219-223. Lancaster: ULMD Publications, University of Lancaster. 
Hanzon, B. and Hut, R. (1991) New results on the projection filter. Proceedings of the First European Control Conference, Grenoble, 1991, Vol. I, pp. 623-628.

Hazewinkel, M., Marcus, S. and Sussmann, H. (1983) Nonexistence of finite dimensional filters for conditional statistics of the cubic sensor problem. Syst. Control Lett., 3, 331-340.

Jacod, J. and Shiryayev, A. (1987) Limit Theorems for Stochastic Processes. Grundlehren math. Wissenschaf., 288. Berlin: Springer-Verlag.

Jazwinski, A. (1970) Stochastic Processes and Filtering Theory. Math. Sci. Engng, 64. New York: Academic Press.

Kagan, A., Linnik, Y. and Rao, C. (1973) Characterization Problems in Mathematical Statistics. New York: Wiley.

Khasminskii, R. (1980) Stochastic Stability of Differential Equations. Alphen aan den Rijn: Sijthoff and Noordhoff.

Kloeden, P. and Platen, E. (1992) Numerical Solution of Stochastic Differential Equations. Applic. Math., 23. New York: Springer-Verlag.

Kulhavý, R. (1990) Recursive nonlinear estimation: a geometric approach. Automatica, 26, 545-555.

Kulhavý, R. (1992) Recursive nonlinear estimation: geometry of a space of posterior densities. Automatica, 28, 313-323.

Kulhavý, R. (1996) Recursive Nonlinear Estimation. A Geometric Approach. Lecture Notes Control Inf. Sci., 216, Berlin: Springer-Verlag.

Kunita, H. (1984) Stochastic differential equations and stochastic flows of diffeomorphisms. In P. Hennequin (ed.), Ecole d'Eté de Probabilités de Saint-Flour XII-1982, pp. 144-303. Lecture Notes Math. 1097. Berlin: Springer-Verlag.

Kushner, H. (1967) Approximations to optimal nonlinear filters. IEEE Trans. Automatic Control, 12, $546-556$.

Lang, S. (1995) Differential and Riemannian Manifolds, 3rd edn. Graduate Texts Math., 160. New York: Springer-Verlag.

Maybeck, P. (1979) Stochastic Models, Estimation, and Control, Vol. 2. Math. Sci. Engng, 141-2. New York: Academic Press.

Murray, M. and Rice, J. (1993) Differential Geometry and Statistics. Monogr. Statist. Appl. Probab., 48. London: Chapman \& Hall.

Pardoux, E. (1991) Filtrage non linéaire et équations aux dérivées partielles stochastiques associées. In P. Hennequin (ed.), Ecole d'Eté de Probabilités de Saint-Flour XIX-1989, pp. 67-163. Lecture Notes Math., 1464. Berlin: Springer-Verlag.

Picard, J. (1986) Nonlinear filtering of one-dimensional diffusions in the case of a high signal-to-noise ratio. SIAM J. Appl. Math., 46, 1098-1125.

Picard, J. (1991) Efficiency of the extended Kalman filter for nonlinear systems with small noise. SIAM J. Appl. Math., 51, 843-885.

Picard, J. (1993) Estimation of the quadratic variation of nearly observed semimartingales with application to filtering. SIAM J. Control Optimization, 31, 494-517.

Rozovskii, B. (1990) Stochastic Evolution Systems. Math. Applic. (Soviet Ser.), 35. Dordrecht: Kluwer. van Schuppen, J. (1979) Stochastic filtering theory: a discussion of concepts, methods, and results. In M. Kohlmann and W. Vogel (eds), Stochastic Control Theory and Stochastic Differential Systems, Bad Honnef, 1979, pp. 209-226. Lecture Notes Control Inf. Sci., 16. Berlin: Springer-Verlag.

Received June 1996 and revised June 1997 